\title{
Acoustic Source Distribution on Aerofoils and its Reconstruction from Far-Field Array Measurements
}

\author{
Fabio Casagrande Hirono ${ }^{\mathrm{a}, *}$, Phillip F. Joseph ${ }^{\mathrm{a}}$, Filippo M. Fazi ${ }^{\mathrm{a}}$ \\ ${ }^{a}$ Institute of Sound and Vibration Research, University of Southampton, Southampton, UK, SO17 1 BJ.
}

\begin{abstract}
This paper is concerned with the acoustic source distribution on aerofoils interacting with a turbulent flow. The source distribution is usually taken to be equal to the pressure jump across the aerofoil, whose behaviour is well understood from classical unsteady aerofoil theory. However, much of this source does not radiate to the far-field. This paper deals with the application of Fourier methods to extract the equivalent source distribution on the aerofoil that radiates perfectly to the far-field. This paper shows that, whilst the actual pressure jump across a flat plate tends to infinity at the leading edge while dropping to zero at the trailing edge (due to the Kutta condition), the equivalent source distribution is more uniform across the aerofoil chord, which explains why the radiation spectrum and single-frequency directivity both exhibit strong oscillations due to interference from coherent sources across the chord. A method for extracting this far-field equivalent source distribution from an array of far-field measurements is proposed in this paper.
\end{abstract}

Keywords: Aeroacoustics, Aerofoil interaction noise, Microphone arrays, Source reconstruction

\section{Introduction}

Aerofoils situated in a turbulent flow radiate broadband noise by scattering its vorticity into sound at the leading edge. This interaction leads to the generation of an unsteady pressure jump across the aerofoil, which may be interpreted as a distribution of dipoles acting in the direction normal to the aerofoil surface [1]. For 5 an idealised flat plate, the form of the solution for the pressure jump across the chord is characterised by the leading edge singularity of the form $X^{-1 / 2}$ with the leading edge located at $X=0$, dropping to zero at the trailing edge, where the Kutta condition is applied. The sound field radiated from this source distribution can then be readily computed using the Green's function solution, since the singularity is readily integrable.

The far-field noise frequency spectrum and single-frequency directivity have distinct oscillatory behaviour $[2,3]$, implying strong interference from sources along the aerofoil chord. This behaviour cannot be readily explained by the dipole distribution described above, whose source strength is highly localized towards

\footnotetext{
${ }^{*}$ Corresponding author

Email addresses: fchirono@gmail.com (Fabio Casagrande Hirono), pf j@isvr.soton.ac.uk (Phillip F. Joseph), filippo.fazi@soton.ac.uk (Filippo M. Fazi) 
the leading edge. The effective source distribution responsible for the far-field radiation must therefore be significantly different from that predicted using classical flat plate theory. One of the objectives of this paper is to quantify this equivalent far-field-radiating source distribution, in order to gain a fundamental understanding into the noise generation process and also to indicate the regions of greatest source strength to assist with the development of noise control treatments. A second objective of this paper is to devise a measurement technique that allows this effective far-field source distribution to be reconstructed from far-field acoustic measurements.

Estimation of the source strength distribution in aerofoils responsible for far-field noise is often made using conventional beamforming and associated phased array measurement techniques, such as deconvolution and source power integration [4-9]. Conventional beamforming traditionally assumes a distribution of incoherent point sources, and is therefore potentially inaccurate for distributed, partially-coherent sources. Source power integration approaches [10] can mitigate some of these limitations by representing all sources within a region of interest by a single synthetic equivalent source - for example, in the form of a linear distribution of incoherent point sources of equal amplitude. As the array main lobe width is generally wider than the actual source coherence length, the original assumption of an incoherent distribution of point sources is considered valid for source integration purposes [10]. However, source integration techniques aim to estimate the overall noise radiated from an extended source, and not to characterise the source distribution itself.

An approach which circumvents some of these difficulties for distributed, partially-coherent sources involves inverting for the cross spectral matrix of a distribution of discrete sources, assuming that the matrix of transfer functions between each assumed point source and observer is known [11-14]. However, the matrix inversion step is computationally intensive and potentially ill-conditioned, which might lead to inaccurate source estimates. A potentially more detailed source estimate can be obtained from near-field acoustic holography [15-17], which focuses on measuring the acoustic field very close to the source, and thus considers both radiating and non-radiating acoustic fields in order to improve the reconstruction resolution and accuracy. As holographic approaches demand a large number of sensors positioned very close to the source, this potentially exposes the microphones to large levels of hydrodynamic flow noise, which can contaminate the measurements and lead to excessive noise amplification in the reconstruction step.

A fundamentally different approach has been proposed by Williams $[18,19]$, who advocated the use of far-field sensors to determine the equivalent far-field-radiating sources on vibrating surfaces and identify "hot spots" (i.e. strong radiators of far-field sound). This method involves back-propagating the far-field pressure to the radiator surface using a Fourier-based representation of the sound radiation in a medium at rest [20]. A variation of this approach for cylindrical sources has been proposed under the name "far-field acoustic holography" [21], in contrast to the above mentioned near-field acoustic holography method [15].

A modification of this Fourier-based far-field method has been proposed by the authors of this paper [22] to include flow convection effects in the propagation and backpropagation steps, and estimate the equivalent 
far-field-radiating source distribution of a deterministic, narrowband planar radiator immersed in a uniformly moving medium. This convection-corrected method was then extended to include the dipole-type radiation and the stochastic behaviour of an aerofoil interacting with a turbulent flow, and used to estimate the equivalent far-field-radiating surface pressure jump over the aerofoil surface [23].

The present paper discusses in detail the surface pressure distribution and cross-spectral density predicted analytically from Amiet's model of the noise radiation due to the interaction of a flat plate with turbulent flow [1], and links this source to its radiated far-field via a Spatial Fourier Transform for both single-gust interaction and stochastic turbulence models containing a continuum of harmonic gust components. This relationship is then inverted to estimate the equivalent far-field-radiating source cross-spectral density, where it is shown that the equivalent sources are more uniformly distributed over the aerofoil chord compared with the actual source distribution and spread beyond the aerofoil surface, which explains the presence of oscillations in the directivity and frequency spectrum. The actual and equivalent source cross-spectral densities are then used to evaluate the size of the coherent source region responsible for far-field radiation. It will be shown that the equivalent source distributions tends towards the actual distributions as frequency increases.

Note that an early version of this paper by the authors can be found in the conference papers [23] and [24]. The current paper is an amalgamation and integration of these two papers, with the discussion of the measurement philosophy greatly expanded. Additional figures have also been added to illustrate more clearly the aerofoil surface pressure distribution and wavenumber spectrum in response to a single-gust excitation, and to compare the original surface pressure PSD magnitude and the equivalent far-field-radiating PSD magnitude in response to an isotropic turbulent flow.

\section{Fourier approach to acoustic far-field radiation due to an aerofoil in turbulent flow}

A flat plate interacting with a turbulent flow has been shown to be acoustically identical to dipole sources distributed over the flat plate normal to its surface $[1,25]$, whose strength equals the unsteady pressure jump across the flat plate. Whilst the variation in dipole strength across the chord is well understood from classical theory, not all of this source distribution radiates to the far-field, with the remainder radiating to the nearfield only. This paper is a fundamental investigation into the equivalent source distribution over the aerofoil that radiates with perfect efficiency to the far-field.

\subsection{Acoustic far-field generated by an aerofoil in flow}

In the analysis that follows we assume a Cartesian coordinate system whose origin is located at the centre of the aerofoil. Consider a flat plate aerofoil in the plane $z_{s}=0$, with chord $c=2 b$ and $\operatorname{span} L=2 d$, assumed to interact with a turbulent flow in the $+x$ direction and Mach number $M_{x}=U_{x} / c_{0}$. The unsteady response at a single frequency $\omega$ can be represented as a continuous distribution of dipole sources over the 
surface of the flat plate with strength $\Delta p\left(\mathbf{r}_{s}, \omega\right)$, where $\Delta p$ is the difference in pressures between the top and bottom surfaces, or pressure "jump", at a point $\mathbf{r}_{s}=\left(x_{s}, y_{s}, 0\right)$ on the aerofoil [1]. Assuming a $\mathrm{e}^{+\mathrm{j} \omega t}$ time dependence, the acoustic field at an observer location $\mathbf{r}=(x, y, z)$ can be calculated as

$$
p(\mathbf{r}, \omega)=\int_{-d}^{+d} \int_{-b}^{+b} \Delta p\left(\mathbf{r}_{s}, \omega\right) \frac{\partial}{\partial z_{s}} G_{U_{x}}\left(\mathbf{r} \mid \mathbf{r}_{s}, \omega\right) \mathrm{d} x_{s} \mathrm{~d} y_{s},
$$

where $G_{U_{x}}\left(\mathbf{r} \mid \mathbf{r}_{s}, \omega\right)$ is the convected Green's function, such that $\partial G_{U_{x}}\left(\mathbf{r} \mid \mathbf{r}_{s}, \omega\right) / \partial z_{s}$ may be interpreted as the acoustic transfer function between a point dipole source located at $\mathbf{r}_{s}$ and an observer located at $\mathbf{r}$ in free-field in the presence of a mean flow:

$$
\frac{\partial}{\partial z_{s}} G_{U_{x}}\left(\mathbf{r} \mid \mathbf{r}_{s}, \omega\right)=\left(\mathrm{j} k+\frac{1}{\bar{r}}\right) \frac{\left(\bar{z}-\bar{z}_{s}\right)}{\beta \bar{r}} \frac{\mathrm{e}^{-\mathrm{j} k_{0} \bar{r}}}{4 \pi \beta^{2} \bar{r}} \mathrm{e}^{\mathrm{j} k_{0} M_{x}\left(\overline{\bar{x}}-\overline{\bar{x}}_{s}\right)},
$$

where the overline represents flow-transformed variables [26]:

$$
\overline{\mathbf{r}}=(\overline{\bar{x}}, \bar{y}, \bar{z})=\left(\frac{x}{\beta^{2}}, \frac{y}{\beta}, \frac{z}{\beta}\right), \quad \bar{r}=\left\|\overline{\mathbf{r}}-\overline{\mathbf{r}}_{s}\right\|, \quad \beta=\sqrt{1-M_{x}^{2}} .
$$

Of particular interest in this paper are observer locations in the geometric far-field, in which case we can make the Fraunhofer approximation,

$$
\bar{r}=\left\|\overline{\mathbf{r}}-\overline{\mathbf{r}}_{s}\right\| \approx\|\overline{\mathbf{r}}\|-\frac{\overline{\mathbf{r}} \cdot \overline{\mathbf{r}}_{s}}{\|\overline{\mathbf{r}}\|} .
$$

Errors related to this approximation in the context of the far-field radiation due to a flat plate interacting with a single vortical gust were investigated by the authors in previous work [24]. Errors were found at high frequencies for observers sufficiently close to the flat plate between the far-field approximation and the exact numerical solution in which near-field effects were included. When the observers were moved further away, both solutions tended to converge. These errors are further elaborated in Appendix A, where it is demonstrated that, for a fixed source-observer distance, the error in the Fraunhofer far-field approximation increases with frequency.

In the geometric far-field, Eq. (2) is approximated as

$$
\frac{\partial}{\partial z_{s}} G_{U_{x}}\left(\mathbf{r} \mid \mathbf{r}_{s}, \omega\right) \approx \frac{\partial}{\partial z_{s}} G_{F F}\left(\mathbf{r} \mid \mathbf{r}_{s}, \omega\right)=\mathrm{j} k_{0} \frac{z}{\sigma_{\mathbf{r}}} \frac{\mathrm{e}^{-\mathbf{j} \hat{\mathbf{k}} \cdot\left(\mathbf{r}-\mathbf{r}_{s}\right)}}{4 \pi \sigma_{\mathbf{r}}},
$$

where $\sigma_{\mathbf{r}}$ is the flow-corrected distance from the observer to the origin given by

$$
\sigma_{\mathbf{r}}=\sqrt{x^{2}+\beta^{2}\left(y^{2}+z^{2}\right)}=\beta^{2}\|\overline{\mathbf{r}}\|,
$$

and $\hat{\mathbf{k}}$ is the acoustic wavenumber vector: 


$$
\begin{aligned}
\hat{\mathbf{k}}\left(\mathbf{r}, M_{x}\right) & =\left(\hat{k}_{x}, \hat{k}_{y}, \hat{k}_{z}\right) \\
& =\left(k_{0}\left(\frac{x-M_{x} \sigma_{\mathbf{r}}}{\beta^{2} \sigma_{\mathbf{r}}}\right), k_{0} \frac{y}{\sigma_{\mathbf{r}}}, k_{0} \frac{z}{\sigma_{\mathbf{r}}}\right) .
\end{aligned}
$$

The wavenumber vector $\hat{\mathbf{k}}\left(\mathbf{r}, M_{x}\right)$, herein called the observer wavenumber, is in the direction of propagation of the wavefronts at the observer location, which in the presence of mean flow differs from the direction of the observer vector $\mathbf{r}$ and instead points from the retarded source location. The far-field approximation allows the effects of convection on the phase of the acoustic wave to be readily expressed in the wavenumber domain, thereby avoiding the use of flow-transformed spatial variables.

In the far-field, Eq. (2) can be replaced by Eq. (5) and the radiation integral of Eq. (1) becomes

$$
p(\mathbf{r}, \omega) \approx \mathrm{j} k_{0} \frac{z}{\sigma_{\mathbf{r}}} \frac{\mathrm{e}^{-\mathrm{j} \hat{\mathbf{k}} \cdot \mathbf{r}}}{4 \pi \sigma_{\mathbf{r}}}\left[(2 \pi)^{2} \widetilde{\Delta p}(\hat{\mathbf{k}}, \omega)\right] .
$$
Transform of the aerofoil surface pressure (defined in the next Section) evaluated at the observer wavenumber $\hat{\mathbf{k}}=\left(\hat{k}_{x}, \hat{k}_{y}\right)$. The acoustic far-field at a particular observer location $\mathbf{r}$ is therefore directly related to the surface pressure at the corresponding observer wavenumber $\hat{\mathbf{k}}(\mathbf{r})$. This relationship essentially diagonalizes the radiation integral, and establishes a one-to-one relationship between a single Fourier component of surface pressure and the radiated field at a particular direction.

\subsection{The spatial Fourier Transform and plane waves}

The diagonalization of the radiation integral described in the previous section is described by the Fourier Transform of the pressure jump $\Delta p\left(x_{s}, y_{s}, \omega\right)$, defined over the plane $z_{s}=0$ as $[20,27]$

$$
\widetilde{\Delta p}\left(k_{x}, k_{y}, \omega\right)=\frac{1}{(2 \pi)^{2}} \int_{-\infty}^{+\infty} \int_{-\infty}^{+\infty} \Delta p\left(x_{s}, y_{s}, \omega\right) \mathrm{e}^{\mathrm{j} k_{x} x_{s}} \mathrm{e}^{\mathrm{j} k_{y} y_{s}} \mathrm{~d} x_{s} \mathrm{~d} y_{s} .
$$

From the dispersion relation for a convected medium $[16,17]$, the wavenumber component $k_{z}$ normal to

the source surface is related to its trace components $k_{x}, k_{y}$ on the source plane by

$$
k_{z}=\sqrt{\left(k_{0}-M_{x} k_{x}\right)^{2}-k_{x}^{2}-k_{y}^{2}} .
$$

For real and positive values of $k_{z}$, Eq. (11) describes half the surface of an ellipsoid, shown in Figure 1 , and denote waves that propagate to the far-field. The intersection of the ellipsoid with the $k_{z}=0$ plane defines an ellipse [16, 17], shown in Figure 1 as a dashed-dotted line. The ellipse and ellipsoid dimensions are proportional to $k_{0}$, and thus to frequency. In the absence of mean flow, the ellipse becomes a circle and the ellipsoid becomes a hemisphere, both of radius $k_{0}$. 


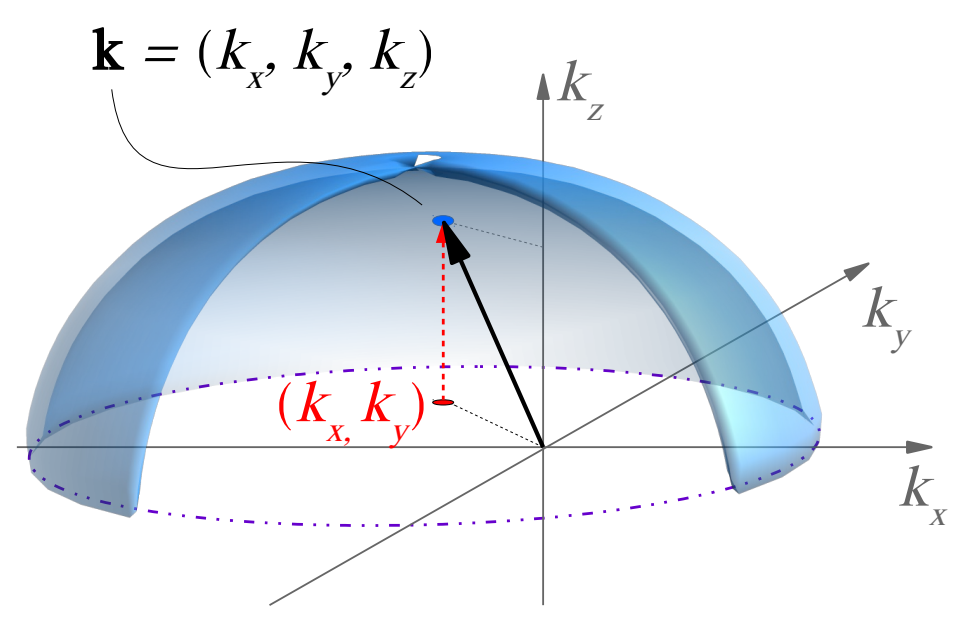

Figure 1: Three-dimensional diagram of the radiation ellipsoid in wavenumber domain. The vector $\mathbf{k}=$ $\left(k_{x}, k_{y}, k_{z}\right)$ is located on the surface of the ellipsoid, and represents a propagating plane wave. The dashdotted line represents the radiation ellipse, located on the $k_{z}=0$ plane.

Wavenumber components $\left(k_{x}, k_{y}\right)$ located inside the ellipse correspond to real values of $k_{z}$, and define acoustic plane waves propagating away from the source and towards a single unique point $\mathbf{r}$ in the farfield, according to Eq. (8). This region is therefore called the "acoustic domain" [28] of the wavenumber spectrum, or "radiation ellipse", and is associated with the main diagonal of the far-field radiation integral operator. Conversely, wavenumber components outside of the ellipse correspond to imaginary values of $k_{z}$, and describe decaying evanescent waves that do not reach the far-field. These evanescent components are thus associated with the off-diagonal terms of the far-field radiation integral operator.

\section{Turbulence-aerofoil interaction noise radiation}

The previous section has established the relationship between far-field noise radiation and the source wavenumber spectrum. We now review the classical flat plate model of Amiet [1] for the prediction of aerofoil-turbulence interaction noise, with emphasis on its wavenumber spectrum to identify the equivalent far-field-radiating source distribution following the principles discussed above.

\subsection{Interaction noise prediction model}

An isotropic turbulent flow is decomposed via Fourier transform in a continuum of streamwise and spanwise vortical (i.e. non-acoustic) wavenumbers $k_{\chi}, k_{\psi}$, and each pair $\left(k_{\chi}, k_{\psi}\right)$ of real-valued wavenumbers represent a single harmonic vortical gust with velocity amplitude $\hat{w}\left(k_{\chi}, k_{\psi}\right)$. Each gust induces a pressure difference $\Delta p$ between the aerofoil surfaces, and all gusts are assumed mutually incoherent. For further details, see, for example, Amiet [1] and Roger [3]. Assuming frozen turbulence, it may be shown that the 
streamwise gust component is $\kappa_{\chi}=\omega / U_{x}$, while the spanwise gust component $k_{\psi}$ takes all real values. Defining $g\left(x_{s}, \kappa_{\chi}, k_{\psi}\right)$ as the non-dimensional pressure jump at any position along the chord $x_{s}$ due to a single velocity gust component $\hat{w}\left(\kappa_{\chi}, k_{\psi}\right)$, the pressure jump due to the continuum of velocity components is given by

$$
\Delta p\left(x_{s}, y_{s}, \omega\right)=2 \pi \rho_{0} \int_{-\infty}^{+\infty} \hat{w}\left(\kappa_{\chi}, k_{\psi}\right) g\left(x_{s}, \kappa_{\chi}, k_{\psi}\right) \mathrm{e}^{-\mathrm{j} k_{\psi} y_{s}} \mathrm{~d} k_{\psi} .
$$

The interaction of an aerofoil with a turbulent in-flow is a stochastic phenomenon. To determine the radiated noise Power Spectral Density therefore the pressure distribution on the surface of the aerofoil must be expressed as the cross-power spectral density

$$
S_{\Delta p \Delta p^{\prime}}\left(\mathbf{r}_{s}, \mathbf{r}_{s}^{\prime}, \omega\right)=\lim _{T \rightarrow \infty}\left[\frac{\pi}{T} \mathrm{E}\left\{\Delta p\left(\mathbf{r}_{s}, \omega\right) \Delta p^{*}\left(\mathbf{r}_{s}^{\prime}, \omega\right)\right\}\right]
$$

Assuming that the incoming turbulence is homogeneous with wavenumber spectral density $\Phi_{w w}\left(\kappa_{\chi}, k_{\psi}\right)$, we may write [1]

$$
U_{x} \delta\left(k_{\psi}-k_{\psi^{\prime}}\right) \Phi_{w w}\left(\kappa_{\chi}, k_{\psi}\right)=\lim _{T \rightarrow \infty}\left[\frac{\pi}{T} \mathrm{E}\left\{\hat{w}\left(\kappa_{\chi}, k_{\psi}\right) \hat{w}^{*}\left(\kappa_{\chi}, k_{\psi}^{\prime}\right)\right\}\right] .
$$

Substituting Eq. (12) into Eq. (13) and integrating over $k_{\psi}^{\prime}$, the surface pressure cross spectrum is of the form

$$
S_{\Delta p \Delta p^{\prime}}\left(\mathbf{r}_{s}, \mathbf{r}_{s}^{\prime}, \omega\right)=\left(2 \pi \rho_{0}\right)^{2} U_{x} \int_{-\infty}^{+\infty} \Phi_{w w}\left(\kappa_{\chi}, k_{\psi}\right) g\left(x_{s}, \kappa_{\chi}, k_{\psi}\right) g^{*}\left(x_{s}^{\prime}, \kappa_{\chi}, k_{\psi}\right) \mathrm{e}^{-\mathrm{j} k_{\psi}\left(y_{s}-y_{s}^{\prime}\right)} \mathrm{d} k_{\psi} .
$$

Finally, combining Eqs. (1) and (15) allows the cross-power spectral density of the radiated pressure field to be expressed as

$$
\begin{aligned}
S_{p p^{\prime}}\left(\mathbf{r}, \mathbf{r}^{\prime}, \omega\right) & =\lim _{T \rightarrow \infty}\left[\frac{\pi}{T} \mathrm{E}\left\{p(\mathbf{r}, \omega) p^{*}\left(\mathbf{r}^{\prime}, \omega\right)\right\}\right] \\
& =\int_{-d}^{+d} \int_{-b}^{+b} \int_{-d}^{+d} \int_{-b}^{+b} S_{\Delta p \Delta p^{\prime}}\left(\mathbf{r}_{s}, \mathbf{r}_{s}^{\prime}, \omega\right) \frac{\partial}{\partial z_{s}} G_{U_{x}}\left(\mathbf{r} \mid \mathbf{r}_{s}, \omega\right) \frac{\partial}{\partial z_{s}^{\prime}} G_{U_{x}}^{*}\left(\mathbf{r}^{\prime} \mid \mathbf{r}_{s}^{\prime}, \omega\right) \mathrm{d} x_{s} \mathrm{~d} y_{s} \mathrm{~d} x_{s}^{\prime} \mathrm{d} y_{s}^{\prime}
\end{aligned}
$$

Equation (16) is completely general and includes near-field effects and the effects of super-critical and subcritical gust components discussed further below. However, it provides no insight into which characteristics of $S_{\Delta p \Delta p^{\prime}}\left(\mathbf{r}_{s}, \mathbf{r}_{s}^{\prime}, \omega\right)$ are responsible for far-field radiation.

This relationship is made explicit by writing $S_{\Delta p \Delta p^{\prime}}\left(\mathbf{r}_{s}, \mathbf{r}_{s}^{\prime}, \omega\right)$ in terms of its wavenumber cross-spectrum $\widetilde{S}_{\Delta p \Delta p^{\prime}}\left(\mathbf{k}, \mathbf{k}^{\prime}, \omega\right)$, which can be obtained by taking a double Spatial Fourier Transform of Eq. (15):

$$
\widetilde{S}_{\Delta p \Delta p^{\prime}}\left(\mathbf{k}, \mathbf{k}^{\prime}, \omega\right)=\frac{1}{(2 \pi)^{4}} \int_{\mathbf{r}_{s}^{\prime}} \int_{\mathbf{r}_{s}} S_{\Delta p \Delta p^{\prime}}\left(\mathbf{r}_{s}, \mathbf{r}_{s}^{\prime}, \omega\right) \mathrm{e}^{\mathrm{j}\left(\mathbf{k} \cdot \mathbf{r}_{s}-\mathbf{k}^{\prime} \cdot \mathbf{r}_{s}^{\prime}\right)} \mathrm{d} \mathbf{r}_{s} \mathrm{~d} \mathbf{r}_{s}^{\prime} .
$$


Using this Fourier transform relationship and noting the far-field approximation of Eq. (5), the far-field noise cross-power spectral density $S_{p p^{\prime}}\left(\mathbf{r}, \mathbf{r}^{\prime}, \omega\right)$ is given by

$$
S_{p p^{\prime}}\left(\mathbf{r}, \mathbf{r}^{\prime}, \omega\right)=k_{0}^{2}\left(\frac{z}{\sigma_{\mathbf{r}}}\right)\left(\frac{z^{\prime}}{\sigma_{\mathbf{r}^{\prime}}}\right) \frac{\mathrm{e}^{-\mathrm{j}\left(\hat{\mathbf{k}} \cdot \mathbf{r}-\hat{\mathbf{k}}^{\prime} \cdot \mathbf{r}^{\prime}\right)}}{(4 \pi)^{2} \sigma_{\mathbf{r}} \sigma_{\mathbf{r}^{\prime}}}(2 \pi)^{4} \widetilde{S}_{\Delta p \Delta p^{\prime}}\left(\hat{\mathbf{k}}, \hat{\mathbf{k}}^{\prime}, \omega\right) .
$$

The pressure PSD, or far-field directivity, $S_{p p}(\mathbf{r}, \omega)$ is a special case for $\mathbf{r}=\mathbf{r}^{\prime}$ (and hence $\hat{\mathbf{k}}=\hat{\mathbf{k}}^{\prime}$ ) and is given by

$$
S_{p p}(\mathbf{r}, \omega)=\left(k_{0} \frac{z}{\sigma_{\mathbf{r}}} \frac{1}{4 \pi \sigma_{\mathbf{r}}}\right)^{2}(2 \pi)^{4} \widetilde{S}_{\Delta p \Delta p}(\hat{\mathbf{k}}, \omega)
$$

This result generalises Eq. (9) to broadband noise, and establishes the unique mapping between a single wavenumber component of pressure on the surface to the radiation at a single far-field observer.

\subsection{Wavenumber analysis of single-gust response functions}

Equation (16) relates the cross spectrum of the far-field acoustic pressure to the cross spectrum of the surface pressure, and thus to the single-gust surface pressure response function $g$. Turbulent gust components can be categorized as either supercritical gusts $\left(\left|k_{\psi}\right|<k_{\psi}^{c r i t}\right)$, for which the flat plate response is an efficient radiator of sound, or subcritical gusts $\left(\left|k_{\psi}\right|>k_{\psi}^{c r i t}\right)$, for which the flat plate response is an inefficient radiator of sound [29]. The span-wise vortical wavenumber $k_{\psi}^{c r i t}$ defines the boundary between these two regimes [29]:

$$
k_{\psi}^{c r i t}=\frac{\kappa_{\chi} M_{x}}{\beta} .
$$

Before proceeding to determine the effective far-field-radiating source distribution, we first investigate the flat plate surface pressure distribution due to the interaction with the turbulent in-flow, as specified by Eq. (15). The basis for this calculation is the single frequency-wavenumber response function $g$, which assumes different forms for super-critical and sub-critical vortical gust components, discussed below. These classical functions have a linearly-varying phase along the chord which, together with the spanwise variation imposed by the gust, are shown to describe a purely acoustic wavenumber in response to a supercritical gust, and a purely evanescent wavenumber in response to a subcritical gust. However, due to spatial windowing effects, a finite aerofoil is shown to respond to either type of gust with a combination of radiating and evanescent behaviour.

Amiet uses Schwarzschild's technique to calculate the surface pressure on the aerofoil [1,30]. This method consists of first computing the analytical solution for a gust impinging at the leading edge of a semi-infinite aerofoil - that is, with the trailing edge at infinity. This is followed by calculating the second-order backscattering at the trailing edge of another semi-infinite aerofoil, this time with the leading edge at infinity, to correct for the finite aerofoil chord and ensure the Kutta condition applies. Further iterations could in 
principle be included, but in practice the first two terms are usually sufficient for most cases [2]. For brevity, this section discusses only the first-order leading edge responses for super- and subcritical gusts. However, all results shown in Figures are calculated using both first- and second-order solutions, and thus include the Kutta condition.

\subsubsection{Super-critical gusts}

The non-dimensional pressure jump $g$ due to a super-critical vortical gust impinging upon the flat plate leading edge has been derived by Graham [29] and Adamczyk [31] from the solution of the acoustic wave equation and appropriate boundary conditions. Adopting the notation due to Reboul [32], the leading-edge scattering term $g$ is given by

$$
g\left(x_{s}, k_{\chi}, k_{\psi}\right)=\frac{1}{\pi \sqrt{\pi\left(x_{s} / b+1\right)\left(k_{\chi} b+\beta^{2} \kappa\right)}} \mathrm{e}^{-\mathrm{j} k_{x}^{\text {sup }}\left(x_{s}+b\right)} \mathrm{e}^{-\mathrm{j} \pi / 4},
$$

where $\mu_{a}=k_{\chi} M_{x} b / \beta^{2}$ and $\kappa$ is given by

$$
\kappa^{2}=\mu_{a}^{2}-\left(\frac{k_{\psi} b}{\beta}\right)^{2}
$$

and $k_{x}^{\text {sup }}$ is the surface pressure response chord-wise acoustic wavenumber

$$
\begin{aligned}
k_{x}^{\text {sup }} & =\frac{1}{b}\left(\kappa-\mu_{a} M\right) \\
& =\frac{1}{\beta^{2}}\left(\sqrt{k_{0}^{2}-\left(k_{\psi} \beta\right)^{2}}-k_{0} M_{x}\right),
\end{aligned}
$$
nent travelling over an infinite plane has an acoustic wavenumber spectrum corresponding to the Dirac Delta function $\delta\left(k_{x}-k_{x}^{s u p}, k_{y}-k_{\psi}\right)$. Its components $\left(k_{x}^{s u p}, k_{\psi}\right)$ are solutions to the acoustic dispersion relation in a convected medium $[16,17]$, confirming that the flat plate response is an acoustic wave travelling parallel 
to the surface and is therefore situated on the radiation ellipse, as indicated by the circles in Figure 2. This plane wave solution may be shown to be travelling at the sound speed $c_{0}$ relative to the moving fluid.

For a normally incident gust, $k_{\psi}=0$, which when substituted into Eq. (24) predicts wavenumber components of the travelling wave given by $\left(k_{x}, k_{y}\right)=\left(k_{x}^{s u p}, 0\right)$, which is situated at the right-most point on the ellipse. In this case, the travelling wave is propagating downstream with phase speed equal to $c_{0}+U_{x}$, and its span-wise phase speed is infinitely large (i.e. there is no span-wise variation). As $\left|k_{\psi}\right|$ increases and the gust becomes more oblique, the span-wise phase velocity becomes slower and Eq. (24) predicts that $k_{x}^{\text {sup }}$ reduces. At the critical span-wise gust wavenumber, $k_{\psi}=k_{\psi}^{c r i t}$, Eq. (24) indicates that the acoustic wavenumber components $\left(k_{x}^{\text {sup }}, k_{\psi}\right)$ are now situated at the uppermost (or lowermost when $k_{\psi}=-k_{\psi}^{\text {crit }}$ ) point on the radiation ellipse, shown by the square markers in Figure 2. At this critical wavenumber, the travelling wave span-wise phase velocity relative to the fluid is equal to the speed of sound $c_{0}$.

As discussed previously, the amplitude of the acoustic travelling wave $\exp \left(-\mathrm{j}\left[k_{x}^{s u p} x_{s}+k_{\psi} y_{s}\right]\right)$ varies significantly across the chord, tending to infinity and zero at the leading edge and trailing edge, respectively. Upstream and downstream of the aerofoil the amplitude is zero, as there is no solid boundary to support the surface pressure jump, and the physical source distribution is therefore bounded by the aerofoil chord and span. Its corresponding wavenumber pressure spectrum must therefore be unbounded, with non-zero values both inside and outside the radiation ellipse. There is therefore a fundamental difference between the physical source distribution and the far-field-radiating source distribution, which was pointed out to be bounded in wavenumber domain and hence must be unbounded in spatial domain.

Representative examples of single-gust surface pressure spatial distributions and their corresponding wavenumber distributions for a normalised frequency of $k_{0} c=5$ and Mach number $M_{x} \approx 0.17$ were calculated using Eqs. (12) and (10), respectively, and are shown in Figures 3a and 3b. Wavenumber components with largest pressure can be seen to be mostly centred around $\left(k_{x}^{s u p}, k_{\psi}\right)$, with contributions occurring in sidelobes situated both within and beyond the acoustic region, leading to both radiating and evanescent behaviour.

\subsubsection{Sub-critical gusts}

Vortical gust wavenumber components exceeding the critical value, $\left|k_{\psi}\right|>k_{\psi}^{\text {crit }}$, whose span-wise phase velocities along the leading edge are subsonic in the reference frame moving with the fluid, are referred as sub-critical gusts. In an idealised aerofoil of infinite span these gusts components are perfectly non-radiating, but radiate weakly for real aerofoils with finite span $[2,3,33]$. The form of the surface pressure response for these components will now be investigated. The leading-edge scattering term $g^{\prime}$ in response to a sub-critical gust is given by

$$
g^{\prime}\left(x_{s}, k_{\chi}, k_{\psi}\right)=\frac{1}{\pi \sqrt{\pi\left(x_{s} / b+1\right)\left(k_{\chi} b-j \beta^{2} \kappa^{\prime}\right)}} \mathrm{e}^{-\mathrm{j} k_{x}^{s u b}\left(x_{s}+b\right)} \mathrm{e}^{-\mathrm{j} \pi / 4},
$$

where $\kappa^{\prime}$ is given by 


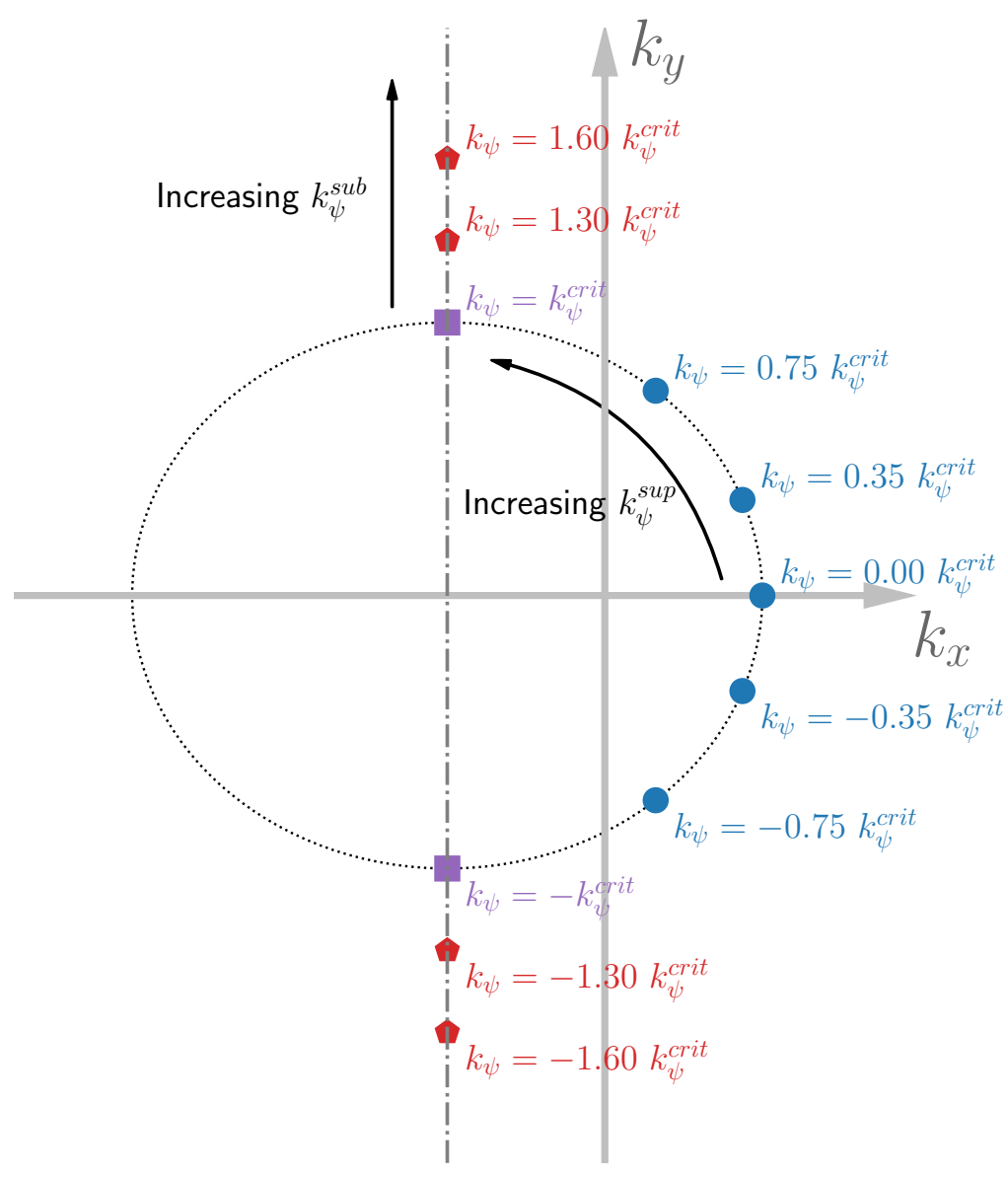

Figure 2: Diagram displaying in acoustic wavenumber space $\left(k_{x}, k_{y}\right)$ the location of the main wavenumber component of the aerofoil response function $g\left(x_{s}, k_{\chi}, k_{\psi}\right)$ for different hydrodynamic spanwise wavenumbers $k_{\psi}$ : supercritical gusts responses are indicated with circles, critical gusts responses are indicated with squares, and subcritical gusts responses are indicated with pentagons. 


$$
\begin{aligned}
\kappa^{\prime 2} & =-\kappa^{2} \\
& =\left(\frac{k_{\psi} b}{\beta}\right)^{2}-\mu_{a}^{2},
\end{aligned}
$$

As for super-critical gusts, the flat plate response to a sub-critical gust also exhibits a $\left(x_{s}+b\right)^{-1 / 2}$ type singularity at the leading edge. This first-order leading-edge scattering term also does not satisfy the Kutta condition by itself.

The acoustic chord-wise wavenumber $k_{x}^{s u b}$ can be written as a sum of real and imaginary parts, as

$$
\begin{aligned}
k_{x}^{s u b} & =\frac{1}{b}\left(-\mu_{a} M_{x}-\mathrm{j} \kappa^{\prime}\right) \\
& =\left(-\frac{k_{0} M_{x}}{\beta^{2}}\right)+\mathrm{j}\left(-\frac{1}{\beta^{2}} \sqrt{\left(k_{\psi} \beta\right)^{2}-k_{0}^{2}}\right),
\end{aligned}
$$

where the real part corresponds to a travelling wave in the downstream direction, and the imaginary part is always negative and corresponds to an exponential decay of the surface pressure jump in the downstream direction. The surface pressure response to sub-critical gusts is therefore mostly concentrated at the leading edge, and rapidly decays towards the trailing edge, as seen on the rightmost entry of Figure 3a. Note that the real component $\operatorname{Re}\left(k_{x}^{s u b}\right)=-k_{0} M_{x} / \beta^{2}$ is independent of $k_{\psi}$, and hence so is the chordwise phase speed. The decay rate is determined from the imaginary component $\operatorname{Im}\left(k_{x}^{s u b}\right)$ and increases with increasing $k_{\psi}$ (i.e. increasing gust obliqueness).

The phase variation over the aerofoil surface is therefore given by $\exp \left(-\mathrm{j}\left[\operatorname{Re}\left(k_{x}^{s u b}\right) x_{s}+k_{\psi} y_{s}\right]\right)$, which on an infinite plane has a wavenumber spectrum corresponding to the Dirac Delta function $\delta\left(k_{x}-\operatorname{Re}\left(k_{x}^{s u b}\right), k_{y}-\right.$ $\left.k_{\psi}\right)$. The Dirac Delta function for critical spanwise wavenumber $\left|k_{\psi}\right|=k_{\psi}^{c r i t}$ is situated at the uppermost/lowermost points of the radiation ellipse. As $\left|k_{\psi}\right|$ increases above this, the position of the Delta function moves vertically away from the radiation ellipse, as indicated by pentagons in Figure 2 . The travelling wave component of the surface pressure described above with unit amplitude everywhere radiates as an evanescent wave in the spanwise direction by virtue of its subsonic spanwise velocity in the moving reference frame.

However, the amplitude of this wave component varies along the chord, as indicated in Eq. (25), and is zero upstream and downstream of the aerofoil. The wavenumber spectrum of the response of a finite aerofoil to a subcritical gust will be unbounded, as seen on the rightmost entry of Figure 3b. As some sidelobes will fall within the radiation ellipse, sub-critical gusts will therefore excite some acoustic wave components that are able to radiate to the far-field. For more oblique gusts, the travelling wave component moves further away from the radiation ellipse, and the contribution to the pressure within the radiation ellipse becomes significantly weaker. Both super-critical and sub-critical gust components in the incoming turbulent flow therefore contribute, to different degrees, to the acoustic far- and near-fields. 

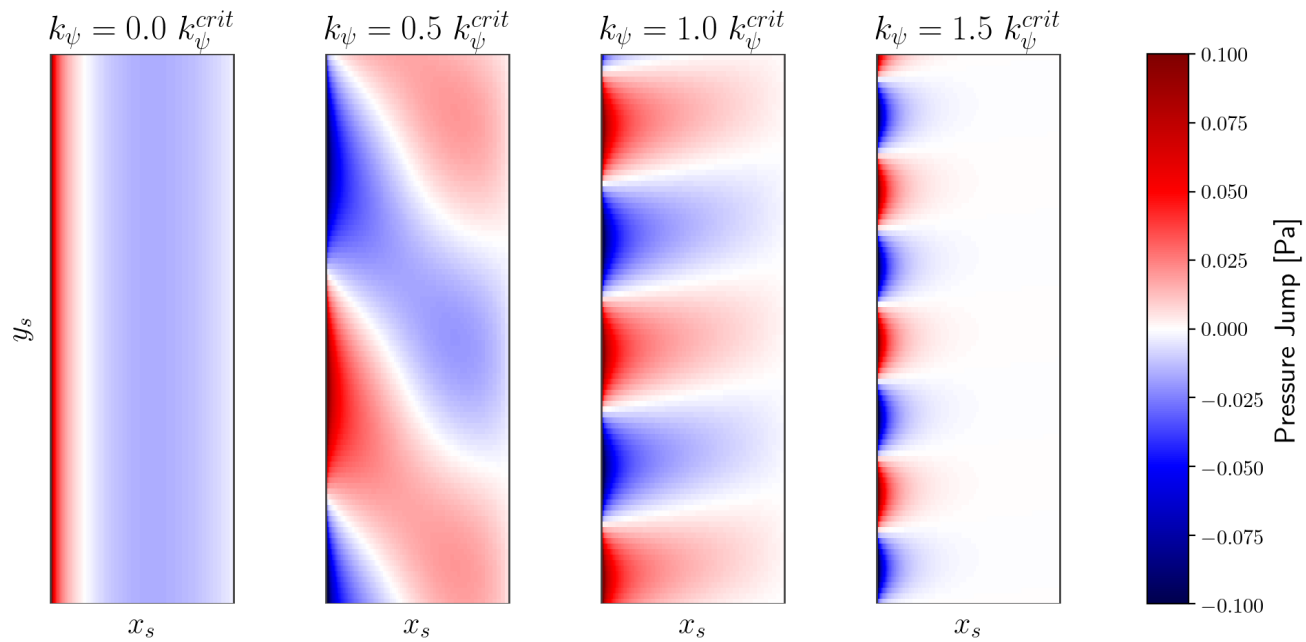

(a)
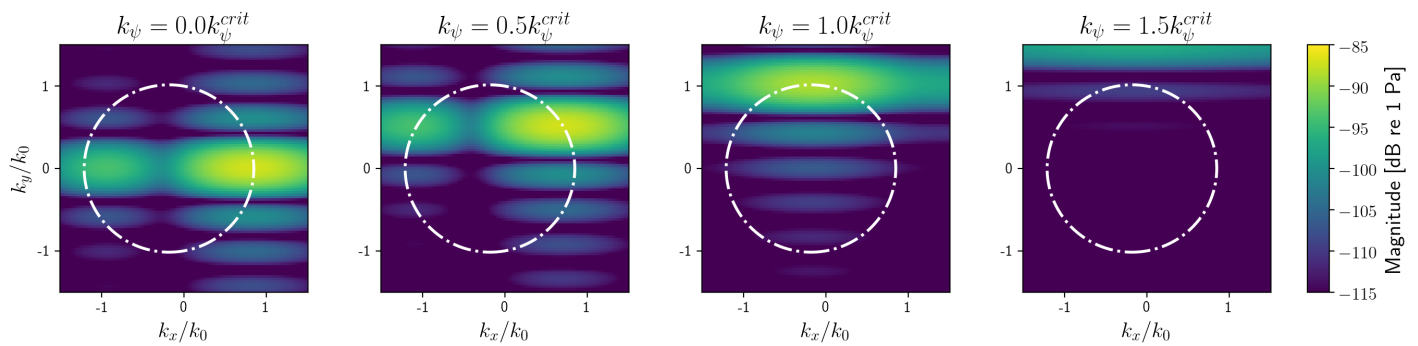

(b)

Figure 3: Typical single-gust aerofoil surface pressure jump for different range of super- and sub-critical gusts $\left(k_{0} c=5, M_{x} \approx 0.17\right)$ : (a) real part of surface pressure jump $\operatorname{Re}\left(\Delta p\left(x_{s}, y_{s}\right)\right)$ (leading edge at the left); (b) pressure jump wavenumber transform magnitude $\left|\widetilde{\Delta p}\left(k_{x}, k_{y}\right)\right|$.

\subsection{Surface pressure wavenumber power spectra}

The previous section has investigated the flat plate response and its wavenumber spectrum at a single frequency to different discrete vortical gust components above and below their critical value. However, turbulent flows comprise a continuum of mutually uncorrelated gust wavenumber components, characterized by its wavenumber spectral density $\Phi_{w w}\left(k_{\chi}, k_{\psi}\right)$. The surface pressure response to an incoming turbulent flow may be obtained by integrating, incoherently, the single-gust response function appropriately weighted by $\Phi_{w w}\left(k_{\chi}, k_{\psi}\right)$. The surface pressure wavenumber auto-spectrum $S_{\Delta p \Delta p}(\mathbf{k})$ is then obtained from Eq. (17) by setting $\mathbf{k}=\mathbf{k}^{\prime}$. For idealised isotropic, homegeneous turbulence with mean square velocity $\overline{w^{2}}$ and integral length-scale $\Lambda$, the von Karman model for $\Phi_{w w}\left(k_{\chi}, k_{\psi}\right)$ is given by [1]

$$
\begin{aligned}
\Phi_{w w}\left(k_{\chi}, k_{\psi}\right) & =\frac{4}{9 \pi} \frac{\overline{w^{2}}}{k_{e}^{2}} \frac{\check{k}_{\chi}^{2}+\check{k}_{\psi}^{2}}{\left(1+\check{k}_{\chi}^{2}+\check{k}_{\psi}^{2}\right)^{7 / 3}}, \\
k_{e} & =\frac{\sqrt{\pi}}{\Lambda} \frac{\Gamma(5 / 6)}{\Gamma(1 / 3)}
\end{aligned}
$$


where $\check{k}_{i}=k_{i} / k_{e}$ and $\Gamma(\cdot)$ is the Gamma function.

The mean square velocity $\overline{w^{2}}$ and integral length-scale $\Lambda$ were quantified by adjusting a theoretical model to give best fit to the velocity spectrum measured on the ISVR's open jet wind tunnel. A turbulence grid was positioned inside the contraction nozzle, thus generating a good approximation to homogeneous, isotropic turbulent flows, and the best fit to the spectrum was obtained for $T_{i}=\sqrt{w^{2} / U_{x}^{2}}=0.025$ and $\Lambda=0.007$ $\mathrm{m}$. The wavenumber power spectral density $\widetilde{S}_{\Delta p \Delta p}(\mathbf{k})$ of the surface pressure response of a flat plate with dimensions of $c=0.15 \mathrm{~m}$ and $d=0.45 \mathrm{~m}$ due to interaction with isotropic turbulence using the above mentioned values was calculated using Eq. (17) and is shown in $\mathrm{dB}$ in the left-hand side of Figure 4, at the three normalized frequencies of $k_{0} c \in[0.5,5,20]$.

At the lowest frequency, $k_{0} c=0.5$, the surface pressure is uniformly distributed over the range of wavenumbers investigated and is almost identical inside and outside the radiation ellipse. It is worth noting that the radiation ellipse dimensions, and hence its area, are comparatively small at such low frequency, and thus only a small portion of the total pressure can be inside the radiation ellipse and hence radiate to the far-field. As most of the surface pressure has wavenumber components outside of the radiation ellipse, the difference between the actual surface pressure distribution across the aerofoil and the equivalent far-field-radiating source distribution is therefore greatest at lower frequencies.

At the higher frequency of $k_{0} c=5$, the surface pressure PSD is more concentrated around the right hand side of the radiation ellipse, forming a crescent shape. To the left of this region is a similar shape of relatively low pressure. More of the surface pressure has wavenumber components within the radiation ellipse and there is now a smaller difference between the actual surface pressure distribution and the equivalent radiating source distribution.

Finally at the highest frequency of interest, $k_{0} c=20$, the surface pressure is even more concentrated around the right hand side of the radiation ellipse, with a number of alternating high and low pressure bands of near-identical profile. The surface pressure is increasingly concentrated around the right hand side of the ellipse as the frequency is increased, suggesting that the equivalent radiating source distribution tends to the physical source distribution in the high frequency limit.

The surface pressure PSD distribution inside the radiation ellipse is linked directly to the far-field directivity $S_{p p}(\mathbf{r})$ via Eq. (19). Each wavenumber pair $\left(k_{x}, k_{y}\right)$ within the acoustic domain only contributes to the far-field pressure at a single observer and makes zero contribution in all other directions. This relationship is indicated in Fig. 1 in which a single surface pressure component with wavenumbers $\left(k_{x}, k_{y}\right)$ maps to a unique propagating plane wave with components $\left(k_{x}, k_{y}, k_{z}\right)$.

To the right of the surface pressure wavenumber spectra in Figure 4 are their corresponding far-field radiation patterns, computed from Eq. (19) for the observer distance of $R=10 L^{2} / \lambda$ to ensure far-field conditions (see Appendix A). These directivity patterns are presented over a hemisphere projected onto the $z=0$ plane. The polar angle $\theta$ is shown along the radius, while the azimuthal angle $\phi$ is measured 
anti-clockwise from $(x / R, y / R)=(1,0)$. The surface pressure wavenumber spectrum within the radiation ellipse is simply a mapping of the far-field directivity to within the dipole directivity factor $z / \sigma_{\mathbf{r}}$, as indicated in Eq. (19). The surface pressure wavenumber spectrum along the right hand side of the ellipse is therefore much greater than its corresponding directivity along the right hand side of the figures, where $\theta \approx 90^{\circ}$ and the dipole directivity is close to zero.

At the lowest frequency $k_{0} c=0.5$, the radiation is omni-directional (to within the factor $z / \sigma_{\mathbf{r}}$ ), which is entirely consistent with the uniform distribution of surface pressure wavenumber components within the radiation ellipse. The aerofoil therefore radiates as a compact dipole in the low frequency limit. At the higher frequency $k_{0} c=5$, the radiation pattern can be seen to comprise a main radiation lobe at about $\theta \approx 34^{\circ}$, with a single minor lobe. Finally, at the highest frequency of interest, $k_{0} c=20$, the main radiation lobe now occurs at a larger polar angle of $\theta \approx 62^{\circ}$ and the number of minor lobes has increased.

These characteristics for aerofoil leading edge noise far-field radiation are well documented in the literature $[3,34,35]$, which provides direct confirmation of the validity of Eq. (19) for linking the surface pressure wavenumber spectrum within the radiation ellipse to the far-field directivity and the dipole directivity factor $z / \sigma_{\mathbf{r}}$. Note that the wavenumber components of greatest pressure on the radiation ellipse are also the most significantly affected by the dipole directivity factor. This effect can be made explicit by representing $z / \sigma_{\mathbf{r}}$ in the wavenumber domain, which from Eq. (8) has the form

$$
\frac{z}{\sigma_{\mathbf{r}}}=\frac{\hat{k}_{z}}{k_{0}}
$$

As $\hat{k}_{z}=0$ is on the radiation ellipse, the region of maximum surface pressure situated on, and close to, the radiation ellipse is weakly radiating. 


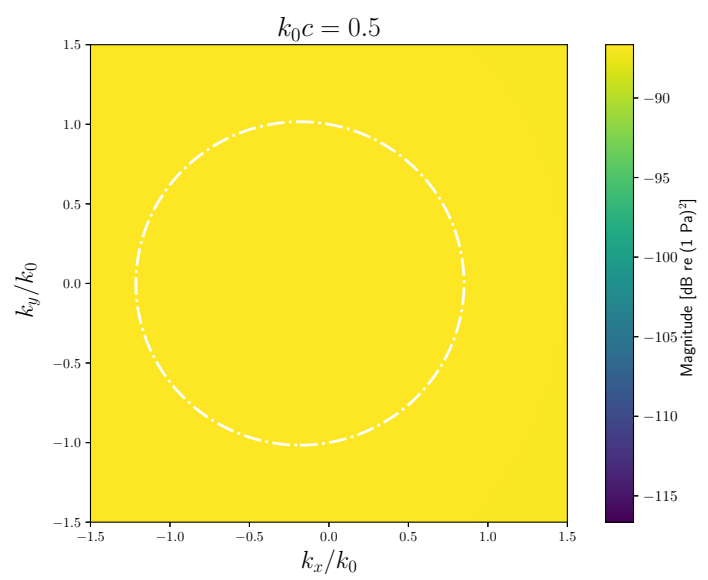

(a)

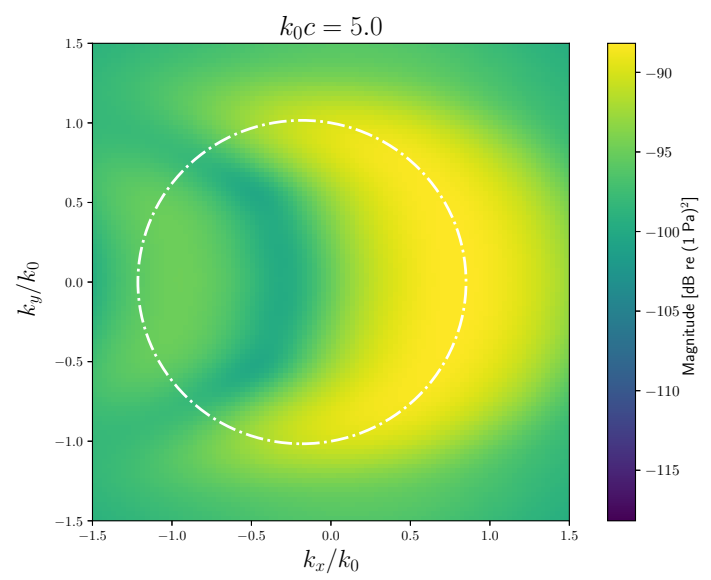

(c)

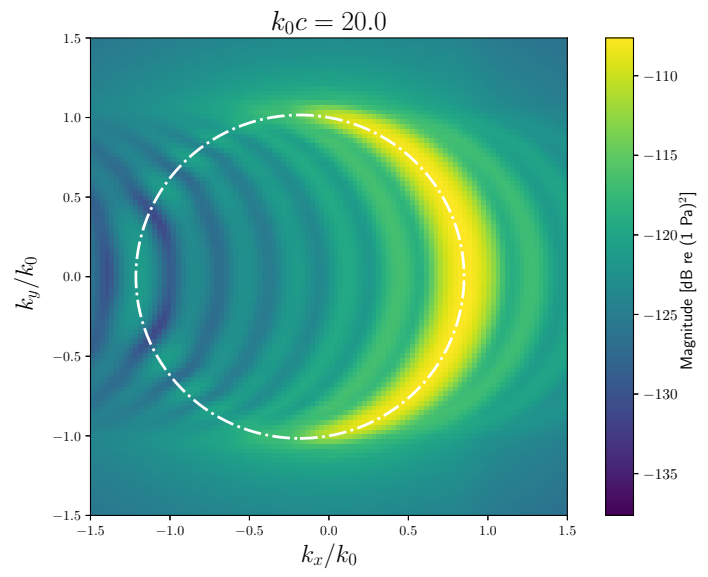

(e)

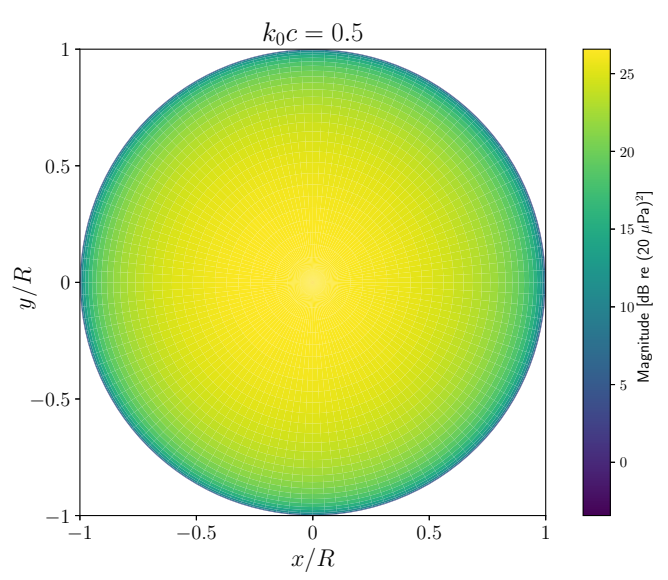

(b)

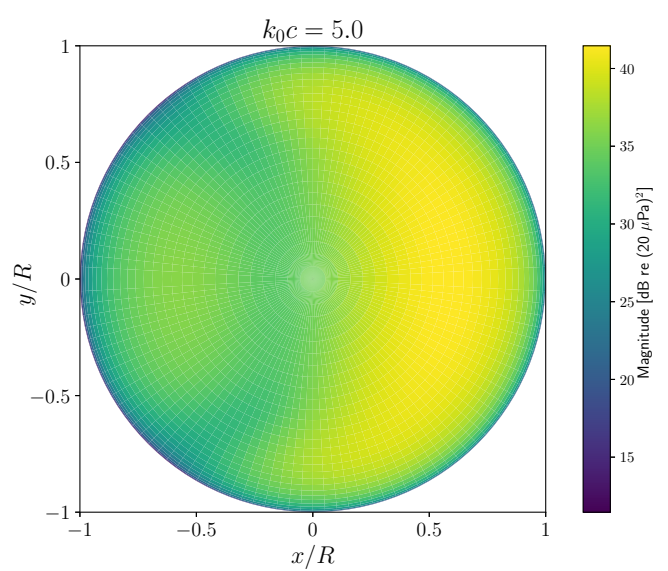

(d)

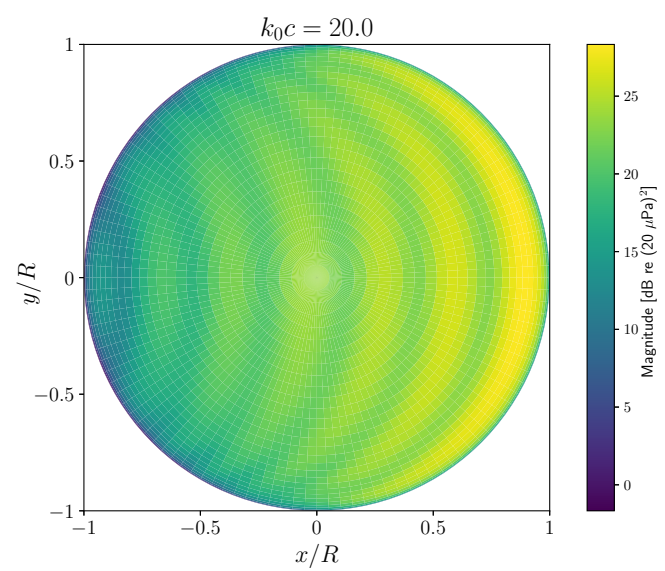

(f)

Figure 4: Left column: surface pressure PSD magnitude $\left|\widetilde{S}_{\Delta p \Delta p}(\mathbf{k})\right|$ (in $\mathrm{dB}$ re $\left.(1 \mathrm{~Pa})^{2}\right)$ in wavenumber domain; right column: far-field directivity $\left|S_{p p}(\mathbf{r})\right|$ (in $\mathrm{dB}$ re $\left.(20 \mu \mathrm{Pa})^{2}\right)$. (a) and (b): low frequency $\left(k_{0} c=0.5\right) ;(\mathrm{c})$ and $(\mathrm{d})$ medium frequency $\left(k_{0} c=5\right) ;(\mathrm{e})$ and $(\mathrm{f})$ : high frequency $\left(k_{0} c=20\right)$. 


\section{Far-field source estimation}

\subsection{Inverse equation for source amplitude}

The previous sections have studied in detail the characteristics of the surface pressure distribution over a flat plate interacting with a turbulent flow. This surface pressure response was represented in the wavenumber domain to allow the separation of propagating wave components within the radiation ellipse from non-propagating (evanescent) pressure components outside the ellipse. A unique and simple mapping was established between surface pressure wavenumber components within the radiation ellipse and the far-field directivity. This relationship is now exploited to develop a measurement procedure by which far-field acoustic measurements from an aerofoil can be used to reconstruct the equivalent far-field-radiating surface pressure distribution.

The starting point of the measurement method is to obtain an estimate of the source wavenumber spectrum $\widetilde{\Delta p_{\text {equiv }}}\left(\hat{k}_{x}, \hat{k}_{y}\right)$ within the radiation ellipse, which can be determined from far-field acoustic measurements by rearranging Eq. (9):

$$
\widetilde{\Delta p_{\text {equiv }}}(\hat{\mathbf{k}}, \omega)=\frac{1}{(2 \pi)^{2}}\left(\mathrm{j} k_{0} \frac{z}{\sigma_{\mathbf{r}}} \frac{\mathrm{e}^{-\mathrm{j} \hat{\mathbf{k}} \cdot \mathbf{r}}}{4 \pi \sigma_{\mathbf{r}}}\right)^{-1} p(\mathbf{r}, \omega) .
$$

The Inverse Fourier Transform of the surface pressure wavenumber spectrum within the radiation ellipse can then be used to estimate the surface pressure distribution $\Delta p_{\text {equiv }}\left(\mathbf{r}_{s}\right)$ that radiates over the far-field hemisphere, which is completely absent of a near field, and has the form

$$
\Delta p_{\text {equiv }}\left(\mathbf{r}_{s}, \omega\right)=\int_{\mathbf{k}} \widetilde{\Delta p_{\text {equiv }}}(\hat{\mathbf{k}}, \omega) \mathrm{e}^{-\mathrm{j} \hat{\mathbf{k}} \cdot \mathbf{r}_{s}} \mathrm{~d} \hat{\mathbf{k}}, \quad \hat{\mathbf{k}} \in \operatorname{Rad} . \text { Ellipse. }
$$

The source strength distribution recovered from Eq. (34) therefore precludes high spatial frequencies (i.e. high magnitude wavenumbers) associated with the evanescent field, and lacks the fine spatial detail of the actual source distribution. More fundamentally, the mapping relationship between a single radiating wavenumber component on the flat plate surface and a point on the far-field hemisphere suggests that the wavenumber distribution of surface pressure determined from the measurements must be bounded within the radiation ellipse. The corresponding equivalent spatial surface pressure distribution must therefore be unbounded and extend beyond the physical limits of the aerofoil. By contrast, source reconstructions obtained from Nearfield Acoustic Holography [15], using pressure measurements very close to the source, include these evanescent components in order to increase the spatial resolution.

The main result of this paper is obtained by combining Eqs. (33) and (34) to allow the equivalent far-field-radiating surface pressure distribution $\Delta p_{\text {equiv }}\left(\mathbf{r}_{s}\right)$ to be deduced via an integration of the acoustic pressure $p(\mathbf{r})$ over the entire far-field hemisphere, here represented by a wavenumber-domain integration over the radiation ellipse: 


$$
\Delta p_{\text {equiv }}\left(\mathbf{r}_{s}, \omega\right)=\frac{1}{(2 \pi)^{2}} \int_{\mathbf{k}} p(\mathbf{r}, \omega)\left(\mathrm{j} k_{0} \frac{z}{\sigma_{\mathbf{r}}} \frac{\mathrm{e}^{-\mathrm{j} \hat{\mathbf{k}} \cdot \mathbf{r}}}{4 \pi \sigma_{\mathbf{r}}}\right)^{-1} \mathrm{e}^{-\mathrm{j} \hat{\mathbf{k}} \cdot \mathbf{r}_{s}} \mathrm{~d} \hat{\mathbf{k}}, \quad \hat{\mathbf{k}} \in \text { Rad. Ellipse. }
$$

In practice, however, the far-field acoustic pressure is sampled by an array of discrete sensors at $M$ locations $\mathbf{r}_{m}, m \in[1 \ldots M]$. The two-dimensional integration over the radiation ellipse in Eq. (35) becomes a weighted sum of the microphone pressures $p\left(\mathbf{r}_{m}\right)$ given by

$$
\Delta p_{\text {equiv }}\left(\mathbf{r}_{s}, \omega\right) \approx \frac{1}{(2 \pi)^{2}} \sum_{m=1}^{M} p\left(\mathbf{r}_{m}, \omega\right)\left[\mathrm{j} k_{0} \frac{z_{m}}{\sigma_{\mathbf{r}_{m}}} \frac{\mathrm{e}^{-j \hat{\mathbf{k}}_{m} \cdot\left(\mathbf{r}_{m}-\mathbf{r}_{s}\right)}}{4 \pi \sigma_{\mathbf{r}_{m}}}\right]^{-1} A_{m},
$$

where the term $A_{m}$ is the area in the wavenumber domain centred around the $m$-th observer wavenumber $\hat{\mathbf{k}}_{m}=\left(k_{x, m}, k_{y, m}\right)$, where $\hat{\mathbf{k}}_{m}\left(\mathbf{r}_{m}\right)$ is a function of the $m$-th microphone position due to the unique mapping relationship of Eq. (8). This formulation allows for arbitrary array sampling of the far-field hemisphere, and can be computed using a modified Voronoi diagram of the wavenumber sampling inside the radiation ellipse [23]. Note that the term in square brackets in Eq. (36) corresponds to the far-field convected dipole transfer function $\partial G_{F F}\left(\mathbf{r}_{m} \mid \mathbf{r}_{s}\right) / \partial z_{s}$ previously shown in Eq. (5).

\subsection{Inverse equation for source cross-spectrum}

The noise radiated from aerofoils in a turbulent stream is a stochastic phenomenon, whose equivalent source strength and radiated acoustic field must be expressed in terms of their cross-spectra, previously defined in Eqs. (13) and (16). Combining these Equations with Eq. (35) provides an estimate of the equivalent far-field-radiating surface pressure cross-spectral density $S_{\Delta p \Delta p^{\prime}, \text { equiv }}\left(\mathbf{r}_{s}, \mathbf{r}_{s}^{\prime}\right)$ from the cross-spectrum of the far-field pressure $S_{p p^{\prime}}\left(\mathbf{r}, \mathbf{r}^{\prime}\right)$ :

$$
\begin{aligned}
& S_{\Delta p \Delta p^{\prime}, \text { equiv }}\left(\mathbf{r}_{s}, \mathbf{r}_{s}^{\prime}, \omega\right) \\
& \quad=\frac{1}{(2 \pi)^{4}} \int_{\mathbf{k}^{\prime}} \int_{\mathbf{k}} S_{p p^{\prime}}\left(\mathbf{r}, \mathbf{r}^{\prime}, \omega\right)\left[\left(\mathrm{j} k_{0} \frac{z}{\sigma_{\mathbf{r}}} \frac{\mathrm{e}^{-\mathbf{j} \hat{\mathbf{k}} \cdot\left(\mathbf{r}-\mathbf{r}_{s}\right)}}{4 \pi \sigma_{\mathbf{r}}}\right)\left(\mathrm{j} k_{0} \frac{z^{\prime}}{\sigma_{\mathbf{r}^{\prime}}} \frac{\mathrm{e}^{-j \hat{\mathbf{k}}^{\prime} \cdot\left(\mathbf{r}^{\prime}-\mathbf{r}_{s}^{\prime}\right)}}{4 \pi \sigma_{\mathbf{r}^{\prime}}}\right)^{*}\right]^{-1} \mathrm{~d} \hat{\mathbf{k}} \mathrm{d} \hat{\mathbf{k}}^{\prime}, \\
& \hat{\mathbf{k}}, \hat{\mathbf{k}}^{\prime} \in \text { Rad. Ellipse. }
\end{aligned}
$$

As for the corresponding single-frequency relation of Eq. (35), the terms in parenthesis are identical to the far-field-approximated convected dipole transfer function from Eq. (5), and the cross-spectrum of the far-field pressure is defined for observers located over the entire hemisphere.

When expressing the integration in Eq. (37) in discrete form, we obtain the cross-spectrum version of Eq. (36), given by 


$$
\begin{aligned}
& S_{\Delta p \Delta p^{\prime}, \text { equiv }}\left(\mathbf{r}_{s}, \mathbf{r}_{s}^{\prime}, \omega\right) \\
& \approx \frac{1}{(2 \pi)^{4}} \sum_{m=1}^{M} \sum_{m^{\prime}=1}^{M} S_{p p^{\prime}}\left(\mathbf{r}_{m}, \mathbf{r}_{m^{\prime}}, \omega\right)\left[\left(\mathrm{j} k_{0} \frac{z_{m}}{\sigma_{\mathbf{r}_{m}}} \frac{\mathrm{e}^{-\mathrm{j} \hat{\mathbf{k}}_{m} \cdot\left(\mathbf{r}_{m}-\mathbf{r}_{s}\right)}}{4 \pi \sigma_{\mathbf{r}_{m}}}\right)\left(\mathrm{j} k_{0} \frac{z_{m^{\prime}}}{\sigma_{\mathbf{r}_{m^{\prime}}}} \frac{\mathrm{e}^{-\mathrm{j} \hat{\mathbf{k}}_{m^{\prime}} \cdot\left(\mathbf{r}_{m^{\prime}}-\mathbf{r}_{s}^{\prime}\right)}}{4 \pi \sigma_{\mathbf{r}_{m^{\prime}}}}\right)^{*}\right]^{-1} \\
& \cdot\left[A_{m} A_{m^{\prime}}\right] .
\end{aligned}
$$

Equation (38) is the main result of this paper and allows the estimation of the equivalent far-fieldradiating surface pressure cross-power spectral density $S_{\Delta p \Delta p^{\prime}, \text { est }}\left(\mathbf{r}_{s}, \mathbf{r}_{s}^{\prime}\right)$ from the far-field microphone array cross-power spectral density $S_{p p^{\prime}}\left(\mathbf{r}_{m}, \mathbf{r}_{m^{\prime}}\right)$.

The source estimation method presented above is closely related to frequency-domain conventional beamforming [4], with the important difference that the transfer functions are assumed to be the far-fieldapproximated dipole from Eq. (5). This relationship has been previously studied in relation to the source distribution of monopole sources in a moving medium [23].

\section{Simulation results}

We now examine the equivalent far-field-radiating source distribution on a flat plate interacting with turbulent flow using the source reconstruction method described above. We consider a setup identical to the experimental conditions typically investigated in the ISVR's open-jet wind tunnel. The flow speed is $U_{x}=60 \mathrm{~m} / \mathrm{s}\left(M_{x} \approx 0.17\right)$, and the wavenumber spectral density of the turbulent velocity field is calculated using the von Karman model (Eq. (30)) with the turbulence intensity and integral length-scale presented previously $\left(T_{i}=0.025\right.$ and $\left.\Lambda=0.007 \mathrm{~m}\right)$.

By way of illustration of the method, 350 microphones were arranged over the far-field hemisphere as shown in Figure 5. A similar array was used in Ref. [23] to beamform the source distribution of a number of coherent monopoles in a uniform mean flow.

The wavenumber-domain areas $A_{m}$ are calculated using a modified Voronoi diagram of the wavenumberdomain samples, and are indicated with the dotted lines on the right hand side of Figure 5. The Voronoi diagram is obtained using the Python package scipy.spatial.Voronoi [36], where the seeds are the wavenumber samples of the microphone array, and the cell areas are numerically calculated from the resulting diagram.

\subsection{Surface pressure PSD}

Figure 6a shows the distribution of the actual surface pressure PSD magnitude $\left|S_{\Delta p \Delta p}\left(x_{s}, y_{s}\right)\right|$ (left hand side), obtained from flat plate theory in Section 3.1, alongside the equivalent far-field-radiating surface pressure PSD magnitude $\left|S_{\Delta p \Delta p \text {,equiv }}\left(x_{s}, y_{s}\right)\right|$ (right hand side), estimated from the far-field measurements using Eq. (38), at the three frequencies of $k_{0} c \in[0.5,5,20]$. A more detailed comparison between the original and the estimated PSD is shown in Figure 7 of the chordwise PSD magnitude distribution, obtained from the 


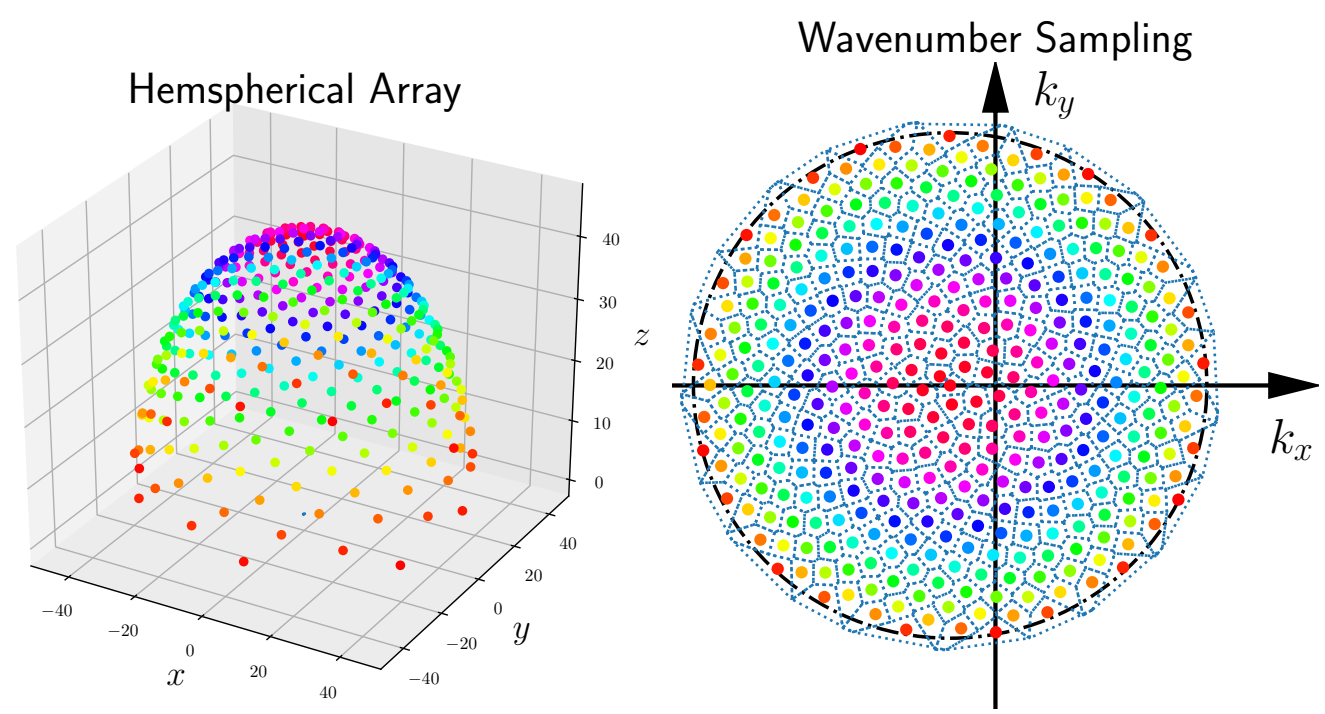

Figure 5: Hemispherical microphone array (left) and corresponding wavenumber-domain sampling (right), with Voronoi cells marked as dotted lines. The $m$-th cell is centred around $\hat{\mathbf{k}}_{m}=\left(\hat{k}_{x, m}, \hat{k}_{y, m}\right)$ and has area $A_{m}$.

same data. Both the leading edge singularity and zero-pressure Kutta conditions are present in the actual surface pressure distribution, plotted as the solid line, and absent in the equivalent source distribution, indicated as a dashed line.

The equivalent far-field-radiating PSDs can be observed to be consistently lower than the actual surface pressure PSDs and extends beyond the physical aerofoil dimensions in both the span and chordwise directions by approximately half of an acoustic wavelength. Similar behaviour has been observed by Williams [19] for radiation problems in a quiescent medium.

In general, the equivalent source varies across the chord much more uniformly than the actual surface pressure, which explains why aerofoil radiation exhibits large oscillations in both the directivity and frequency spectrum due to interference across the chord. Both the leading edge singularity and zero-pressure Kutta conditions are absent in the equivalent source distribution. At low frequencies, the equivalent farfield-radiating source distribution is almost uniform over the aerofoil surface and beyond. As the frequency is increased, the equivalent source distribution becomes concentrated around the aerofoil physical limits. As the frequency is increased further the equivalent and actual source distributions converge, as their radiating wavenumber components become increasingly concentrated within the radiation ellipse. One may therefore argue that aerofoil radiation becomes increasingly efficient as frequency is increased.

In the low frequency case, the estimated source strength is significantly lower than the original by more than $20 \mathrm{~dB}$ at mid-chord. As previously discussed in Section 3.3, this is due to the wavenumber-domain radiating region being comparatively small at this frequency and therefore containing only a small portion of 
the total pressure. Note in Figure 4a the relatively large pressure amplitudes of the evanescent wavenumber components at this frequency.

At higher frequencies the actual and estimated source distributions are in closer agreement in the aft sections of the aerofoil, indicating these regions are significant contributors to the aerofoil far-field sound. The leading edge singularity is again not prominent in the estimated source.

\subsection{Surface pressure coherence}

Finally, we compare the behaviour of the coherence function $\gamma^{2}\left(\mathbf{r}_{s}, \mathbf{r}_{r e f}\right)$ of the equivalent far-fieldradiating surface pressure against the actual surface pressure, which includes non-radiating hydrodynamic components. The coherence function is defined as

$$
\gamma^{2}\left(\mathbf{r}_{s}, \mathbf{r}_{r e f}, \omega\right)=\frac{\left|S_{\Delta p \Delta p^{\prime}}\left(\mathbf{r}_{s}, \mathbf{r}_{r e f}, \omega\right)\right|^{2}}{S_{\Delta p \Delta p}\left(\mathbf{r}_{s}, \omega\right) S_{\Delta p \Delta p}\left(\mathbf{r}_{r e f}, \omega\right)}
$$

Sources with large coherent regions (i.e. $\gamma^{2} \approx 1$ ) compared to the acoustic wavelength $\lambda_{0}$ tend to generate strongly interfering sound fields, characterised by strong oscillations in the frequency spectrum and directivity, and are also more efficient radiators of sound.

Figure 8 shows the surface pressure coherence function $\gamma^{2}\left(\mathbf{r}_{s}, \mathbf{r}_{r e f}\right)$ at the three frequencies investigated at all points on the flat plate relative to a single reference point at mid-span and $10 \%$ of the chord downstream of the leading edge. The Figure shows both the original (left) and the equivalent far-field-radiating (right) source. The actual source distribution is observed to have a short coherence length in both directions, which potentially indicates a relatively inefficient radiation of sound to the far-field. On the other hand, the equivalent far-field-radiating sources have a much larger coherence length in both directions (proportional to $\lambda_{0}$ ). This difference is explained by the contribution of sub-critical gusts to the original surface pressure, as these tend to de-correlate the surface pressure while simultaneously being inefficient sound radiators. Note that at the highest frequency investigated the coherence lengths are comparable on both sources, once again indicating that at these frequencies the original and the equivalent far-field-radiating surface pressure distributions are in closer agreement.

\section{Conclusions}

This paper is an investigation into the equivalent (dipole) source distribution due to an aerofoil interacting with a turbulent flow that radiates perfectly to the far-field. This source distribution is fundamentally different from the actual distribution predicted from classical unsteady aerodynamic theory, in which the pressure jump across the aerofoil exhibits a $X^{-1 / 2}$ type singularity at the leading edge $(X=0)$ and zero pressure jump at the trailing edge due to the Kutta condition. The equivalent source distribution is shown to vary much more uniformly across the chord and extend beyond the physical limits of the aerofoil. The 
equivalent and actual pressure jump across the aerofoil are demonstrated to deviate significantly at low frequencies, but approach each other in both magnitude and coherence as frequency is increased.

This paper has provided a new interpretation of the radiation from aerofoils in a turbulent flow using a Fourier approach adapted to include the effects of a uniform mean flow. In this formulation, a single wavenumber component of the pressure jump situated within the radiation ellipse is purely acoustic, and is shown to radiate to a single far-field observer. This is the basis for a measurement technique proposed in this paper, in which an array of pressure sensors distributed over a far-field hemisphere can be used to determine the complete radiating wavenumber spectrum, which by Fourier Transformation can be used to determine the equivalent far-field-radiating source distribution. Identifying the source distribution may be useful in the design of low-noise aerofoils.

\section{Acknowledgments}

Fabio Casagrande Hirono would like to thank the National Council for Scientific and Technical Development (Conselho Nacional de Desenvolvimento Científico e Tecnológico - CNPq) of the Federal Government of Brazil for sponsoring his PhD degree under the "Science Without Borders" program.

\section{Appendix A. The Fraunhofer far-field approximation}

This Appendix presents how to derive the Fraunhofer far-field approximation, describes the far-fieldapproximated forms for monopole and dipole sources in a mean flow, and discusses the errors committed in this approximation. The derivations herein have been previously presented in Refs. [24, 37].

Extended sources, such as aerofoils, are often modelled using distributions of point sources. As a preliminary step, consider one such point monopole source in a convected medium, with its location $\mathbf{r}_{s}$ close to but not exactly at the origin. The convected Green's function $G_{U_{x}}\left(\mathbf{r} \mid \mathbf{r}_{s}, \omega\right)$, denoting the transfer function between this point monopole and an observer at $\mathbf{r}$, at frequency $\omega$, is written as

$$
G_{U_{x}}\left(\mathbf{r} \mid \mathbf{r}_{s}, \omega\right)=\frac{\mathrm{e}^{-\mathrm{j} k_{0} \bar{r}}}{4 \pi \beta^{2} \bar{r}} \mathrm{e}^{\mathrm{j} k_{0} M_{x}\left(\overline{\bar{x}}-\overline{\bar{x}}_{s}\right)},
$$

where the overline denotes the flow-transformed variables described in Eq. (3). Setting $M_{x}=0$ yields the in the transfer function between a point dipole source at $\mathbf{r}_{s}$, with its acoustic axis in the $+z_{s}$ direction, and an observer at $\mathbf{r}$, presented in Eq. (2).

The convected monopole transfer function in Eq. (A.1) has dependencies on the exact flow-transformed source-to-observer distance $\bar{r}=\left\|\overline{\mathbf{r}}-\overline{\mathbf{r}}_{s}\right\|$ that we would like to approximate for a distant observer. The term in the denominator describes an amplitude decay as a function of distance, and can be reasonably approximated as 


$$
\frac{1}{\beta^{2} \bar{r}} \approx \frac{1}{\sigma_{\mathbf{r}}}
$$

where $\sigma_{\mathbf{r}}$ denotes a convection-corrected observer distance introduced in Eq. (6).

However, the complex exponential $\mathrm{e}^{-\mathrm{j} k_{0} \bar{r}}$ is an oscillating function with range $\bar{r}$, and requires a more accurate approximation. The range $\bar{r}$ is rewritten as

$$
\bar{r}=\|\overline{\mathbf{r}}\|\left(1-2 \frac{\overline{\mathbf{r}} \cdot \overline{\mathbf{r}}_{s}}{\|\overline{\mathbf{r}}\|^{2}}+\frac{\left\|\overline{\mathbf{r}}_{s}\right\|^{2}}{\|\overline{\mathbf{r}}\|^{2}}\right)^{1 / 2} .
$$

It is now assumed that the observer is many source lengths away from the origin, which allows one to approximate $\left\|\overline{\mathbf{r}}_{s}\right\|^{2} /\|\overline{\mathbf{r}}\|^{2} \approx 0$. The resulting expression is written as

$$
\bar{r} \approx\|\overline{\mathbf{r}}\|(1-\alpha)^{1 / 2}, \quad \alpha=2 \frac{\overline{\mathbf{r}} \cdot \overline{\mathbf{r}}_{s}}{\|\overline{\mathbf{r}}\|^{2}} .
$$

The square root term can be expanded using the binomial series $[38,39]$ as

$$
(1-\alpha)^{1 / 2}=1-\frac{\alpha}{2}-\frac{\alpha^{2}}{8}-\ldots,
$$

where the first two terms of the series are retained to become the far-field-approximated distance:

$$
\bar{r} \approx\|\overline{\mathbf{r}}\|\left(1-\frac{\alpha}{2}\right)=\|\overline{\mathbf{r}}\|-\frac{\overline{\mathbf{r}} \cdot \overline{\mathbf{r}}_{s}}{\|\overline{\mathbf{r}}\|}
$$

Equation A.6 is widely used in acoustics [20,39, 40] and electromagnetics [38, 41], but is not often given a name. In the optics community, however, it is known as the Fraunhofer far-field approximation [42].

The far-field-approximated version of the convected Green's function (Eq. (A.1)) is

$$
\begin{aligned}
G_{F F}\left(\mathbf{r} \mid \mathbf{r}_{s}, \omega\right) & =\frac{\mathrm{e}^{-\mathrm{j} k_{0}\|\overline{\mathbf{r}}\|} \mathrm{e}^{\mathrm{j} k_{0} \frac{\overline{\bar{r}} \cdot \overline{\mathbf{r}}_{s}}{\|\bar{r}\|}}}{4 \pi \sigma_{\mathbf{r}}} \mathrm{e}^{\mathrm{j} k_{0} M_{x}\left(\overline{\bar{x}}-\overline{\bar{x}}_{s}\right)} \\
& =\frac{\mathrm{e}^{-\mathrm{j} \hat{\mathbf{k}} \cdot\left(\mathbf{r}-\mathbf{r}_{s}\right)}}{4 \pi \sigma_{\mathbf{r}}},
\end{aligned}
$$

where $\hat{\mathbf{k}}$ is the observer wavenumber vector introduced in Eq. (8).

When compared to the convected monopole, the extra terms in the convected dipole transfer function (Eq. (2)) can be approximated in the far-field as

$$
\left(j k_{0}+\frac{1}{\bar{r}}\right) \frac{\left(\bar{z}-\bar{z}_{s}\right)}{\beta \bar{r}} \approx j k_{0} \frac{z}{\sigma_{\mathbf{r}}},
$$

where the dipole near-field term $1 / \bar{r}$ is ignored and the far-field directivity is expressed as $z / \sigma_{\mathbf{r}}$. The remaining terms in the dipole transfer function are approximated as for the convected monopole, and the final convected dipole far-field transfer function is 


$$
\frac{\partial}{\partial z_{s}} G_{F F}\left(\mathbf{r} \mid \mathbf{r}_{s}, \omega\right)=\mathrm{j} k_{0}\left(\frac{z}{\sigma_{\mathbf{r}}}\right) \frac{\mathrm{e}^{-\mathrm{j} \hat{\mathbf{k}} \cdot\left(\mathbf{r}-\mathbf{r}_{s}\right)}}{4 \pi \sigma_{\mathbf{r}}},
$$

as previously expressed in Eq. (5).

The third term of the binomial series in Eq. (A.5) can be interpreted as an estimate of the error committed when making the Fraunhofer approximation. Its absolute value is

$$
\begin{aligned}
\left|\bar{r}_{\text {error }}\right| & =\|\overline{\mathbf{r}}\|\left(\frac{\alpha^{2}}{8}\right)=\frac{\|\overline{\mathbf{r}}\|}{8}\left(2 \frac{\overline{\mathbf{r}} \cdot \overline{\mathbf{r}}_{s}}{\|\overline{\mathbf{r}}\|^{2}}\right)^{2} \\
& =\frac{1}{2\|\overline{\mathbf{r}}\|}\left\|\overline{\mathbf{r}}_{s}\right\|^{2} \cos ^{2}(\zeta),
\end{aligned}
$$

where $\zeta$ is the angle between $\overline{\mathbf{r}}$ and $\overline{\mathbf{r}}_{s}$.

For low Mach number flow $\left(M_{x} \approx 0\right)$, it can be assumed that $\|\overline{\mathbf{r}}\| \approx\|\mathbf{r}\|=R$ and $\left\|\overline{\mathbf{r}}_{s}\right\| \approx\left\|\mathbf{r}_{s}\right\|$. The error magnitude $\left|\bar{r}_{\text {error }}\right|$ is maximized when $\left\|\overline{\mathbf{r}}_{s}\right\|$ is half of the largest source dimension $L$ (i.e. $\left\|\overline{\mathbf{r}}_{s}\right\|_{\max }=L / 2$ ) and $\cos ^{2}(\zeta)=1$, leading to

$$
\left|\bar{r}_{\text {error }}\right|_{\max }=\frac{L^{2}}{8 R} .
$$

In the electromagnetics literature [38, 41], the Fraunhofer approximation is assumed to be accurate for $\left|r_{\text {error }}\right|_{\max }<\lambda / 16$, resulting in the well-known expression for the geometrical far-field:

$$
R>\frac{2 L^{2}}{\lambda}
$$

Note that the inequality in Equation (A.14) does not denote a hard transition between the near- and farfields, and must be interpreted only as an indication of when the Fraunhofer geometric far-field approximation might be appropriate. Indeed, for a fixed observer distance $R$, the error committed in the Fraunhofer approximation is frequency-dependent, and will be within the suggested tolerance of $\lambda / 16$ for frequencies $f$ such that

$$
f<\frac{R c_{0}}{2 L^{2}}
$$

Hence, the Fraunhofer approximation for a fixed observer distance becomes less accurate as frequency increases.

\section{References}

[1] R. K. Amiet, Acoustic radiation from an airfoil in a turbulent stream, Journal of Sound and Vibration 41, No. 4 (1975) $407-420$.

[2] M. Roger, S. Moreau, Extensions and limitations of analytical airfoil broadband noise models, International Journal of Aeroacoustics 9 (3) (2010) 273-305. 
[3] M. Roger, Broadband noise from lifting surfaces: Analytical modeling and experimental validation, in: R. Camussi (Ed.), Noise Sources in Turbulent Shear Flows: Fundamentals and Applications, Springer-Verlag Wien, 2013.

[4] P. Sijtsma, Phased array beamforming applied to wind tunnel and fly-over tests, Tech. Rep. NLR-TP-2010-549, National Aerospace Laboratory (NLR) - the Netherlands (2010).

[5] T. Biedermann, P. Czeckay, T. Geyer, F. Kameier, C. Paschereit, Noise source identification of aerofoils subjected to leading edge serrations using phased array beamforming, in: 24th AIAA/CEAS Aeroacoustics Conference, 2018, AIAA Paper AIAA 2018-3794.

[6] G. Bampanis, M. Roger, D. Ragni, F. Avallone, C. Teruna, Airfoil-turbulence interaction noise source identification and reduction by leading-edge serrations, in: 25th AIAA/CEAS Aeroacoustics Conference, 2019, AIAA Paper 2019-2741.

[7] R. Merino-Martínez, P. Sijtsma, M. Snellen, T. Ahlefeldt, J. Antoni, C. Bahr, D. Blacodon, D. Ernst, A. Finez, S. Funke, T. Geyer, S. Haxter, G. Herold, X. Huang, W. Humphreys, Q. Leclère, A. Malgoezar, U. Michel, T. Padois, A. Pereira, C. Picard, E. Sarradj, H. Siller, D. Simons, C. Spehr, A review of acoustic imaging methods using phased microphone arrays, CEAS Aeronautical Journal 10 (2019) 197-230. doi:10.1007/s13272-019-00383-4.

[8] G. Yakhina, M. Roger, A. Finez, S. Bouley, V. Baron, S. Moreau, J. Giez, Localization of swept free-tip airfoil noise sources by microphone array processing, AIAA Journal 58 (8) (2020) 1-12. doi:10.2514/1.J058231.

[9] R. Zamponi, S. Satcunanathan, S. Moreau, D. Ragni, M. Meinke, W. Schröder, C. Schram, On the role of turbulence distortion on leading-edge noise reduction by means of porosity, Journal of Sound and Vibration 485 (2020) 1-22.

[10] R. Merino-Martinez, P. Sijtsma, A. R. Carpio, R. Zamponi, S. Luesutthiviboon, A. M. Malgoezar, M. Snellen, C. Schram, D. G. Simons, Integration methods for distributed sound sources, International Journal of Aeroacoustics 18 (4-5) (2019) $444-469$

[11] Y. Kim, P. Nelson, Spatial resolution limits for the reconstruction of acoustic source strength by inverse methods, Journal of Sound and Vibration 265 (2003) 583-608.

[12] T. Suzuki, $l_{1}$ generalized inverse beam-forming algorithm resolving coherent/incoherent, distributed and multipole sources, Journal of Sound and Vibration 330 (2011) 5835-5851.

[13] K. Holland, P. Nelson, An experimental comparison of the focused beamformer and the inverse method for the characterisation of acoustic sources in ideal and non-ideal acoustic environments, Journal of Sound and Vibration 331 (2012) 4425-4437.

[14] K. Holland, P. Nelson, The application of inverse methods to spatially-distributed acoustic sources, Journal of Sound and Vibration 332 (2013) 5727-5747.

[15] J. D. Maynard, E. G. Williams, Y. Lee, Nearfield acoustic holography: I. theory of generalized holography and the development of nah, Journal of the Acoustical Society of America 78 (1985) 1395-1413.

[16] H.-S. Kwon, Y. Niu, Y.-J. Kim, Planar nearfield acoustical holography in moving fluid medium at subsonic and uniform velocity, Journal of the Acoustical Society of America 126 (2010) 1823-1832.

[17] H. Parisot-Dupuis, F. Simon, E. Piot, F. Micheli, Non-intrusive planar velocity-based nearfield acoustic holography in moving fluid medium, Journal of the Acoustical Society of America 133 (2013) 4087-4097.

[18] E. Williams, Supersonic acoustic intensity, Journal of the Acoustical Society of America 97 (1) (1995) $121-127$.

[19] E. Williams, Supersonic acoustic intensity on planar sources, Journal of the Acoustical Society of America 104 $2845-2850$.

[20] E. Williams, Fourier Acoustics: Sound Radiation and Nearfield Acoustical Holography, Academic Press, London, UK, 1999.

[21] A. Norris, Far-field acoustic holography onto cylindrical surfaces using pressure measured on semicircles, Journal of the Acoustical Society of America 102 (4) (1997) 2098-2017.

[22] F. Casagrande Hirono, P. Joseph, F. Fazi, Wavenumber-domain method for source reconstruction from far-field array measurements in flow, in: 7th Berlin Beamforming Conference, 2018, BeBeC Paper BeBeC-2018-D10.

[23] F. Casagrande Hirono, P. Joseph, F. Fazi, Aerofoil surface pressure reconstruction from far-field array measurements, in: 24th AIAA/CEAS Aeroacoustics Conference, 2018, AIAA Paper 2018-3135.

570 [24] F. Casagrande Hirono, P. Joseph, F. Fazi, Aerofoil source estimation from nearfield array measurements, in: 23rd AIAA/CEAS Aeroacoustics Conference, 2017, AIAA Paper 2017-4178.

[25] N. Curle, The influence of solid boundaries upon aerodynamic sound, Proceedings of the Royal Society A 231 (1955) $505-514$.

[26] C. J. Chapman, Similarity variables for sound radiation in a uniform flow, Journal of Sound and Vibration 233, No. 1 (2000) 157-164.

[27] D. Johnson, D. Dudgeon, Array Signal Processing, Prentice-Hall, New Jersey, USA, 1993.

[28] S. Haxter, C. Spehr, Infinite beamforming: Wavenumber decomposition of surface pressure fluctuations, in: 5th Berlin Beamforming Conference, 2014, BeBeC Paper BeBeC-2014-04.

[29] J. Graham, Similarity rules for thin aerofoils in non-stationary subsonic flows, Journal of Fluid Mechanics 43, part 4 (1970) 753-766.

[30] R. K. Amiet, High frequency thin-airfoil theory for subsonic flow, AIAA Journal 14, No. 8 (1976) 1076-1082.

[31] J. Adamczyk, The passage of an infinite swept airfoil through an oblique gust, Tech. Rep. NASA Contractor Report CR-2395, National Aeronautics and Space Administration (NASA) - USA (1974).

[32] G. Reboul, Modélisation du bruit à large bande de soufflante de turboréacteur, PhD Thesis, Laboratoire de Mécanique des Fluides et d'Acoustique - École Centrale de Lyon, Lyon - France (2010).

[33] G. Grasso, J. Christophe, C. Schram, T. Verstraete, Influence of the noise prediction model on the aeroacoustic optimization of a contra-rotating fan, in: 20th AIAA/CEAS Aeroacoustics Conference, 2014, AIAA Paper AIAA $2014-2611$.

[34] V. Clair, C. Polacsek, T. L. Garrec, G. Reboul, M. Gruber, P. Joseph, Experimental and numerical investigation of 
turbulence-airfoil noise reduction using wavy edges, AIAA Journal 51 (11) (2013) 2695-2713.

590 [35] L. D. Santana, C. Schram, W. Desmet, Low-frequency extension of amiet's theory for compact airfoil noise predictions, Journal of Sound and Vibration 372 (23) (2016) 342-356.

[36] E. Jones, T. Oliphant, P. Peterson, et al., SciPy: Open source scientific tools for Python, Available online: http://www.scipy.org/, accessed 23 April 2020 (2001).

[37] F. Casagrande Hirono, Far-field microphone array techniques for acoustic characterisation of aerofoils, PhD Thesis, Institute of Sound and Vibration Research - University of Southampton, Southampton - UK (2018).

[38] T. Sarkar, M. Salazar-Palma, E. Mokole, Physics of Multiantenna Systems and Broadband Processing, John Wiley and Sons, New Jersey, USA, 2008

[39] P. Nelson, Source identification and location, in: F. Fahy, J. Walker (Eds.), Advanced Applications in Acoustics, Noise and Vibration, Spon Press, 2004

0] L. Kinsler, A. Frey, A. Coppens, J. Sanders, Fundamentals of Acoustics, John Wiley and Sons, London, UK, 2000.

[41] W. Stutzman, G. Thiele, Antenna Theory and Design, John Wiley and Sons, New Jersey, USA, 1998.

[42] J. Goodman, Introduction to Fourier Optics, Roberts \& Company, Colorado, USA, 2005. 


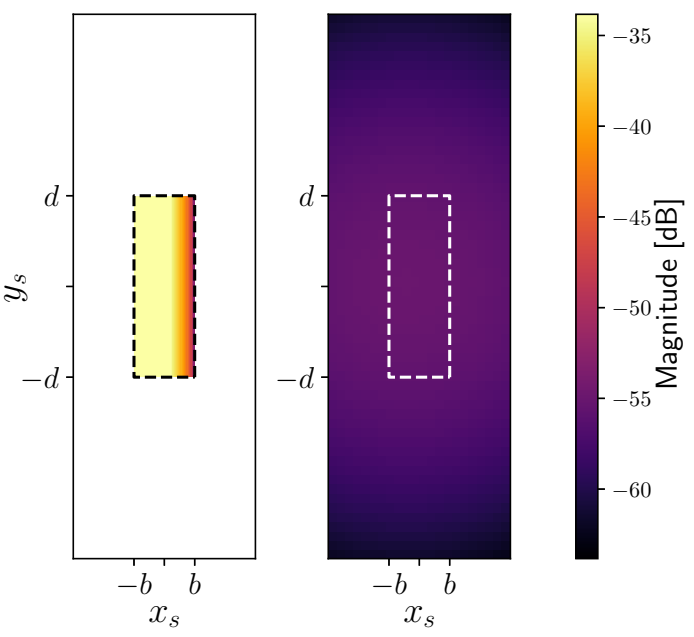

(a)
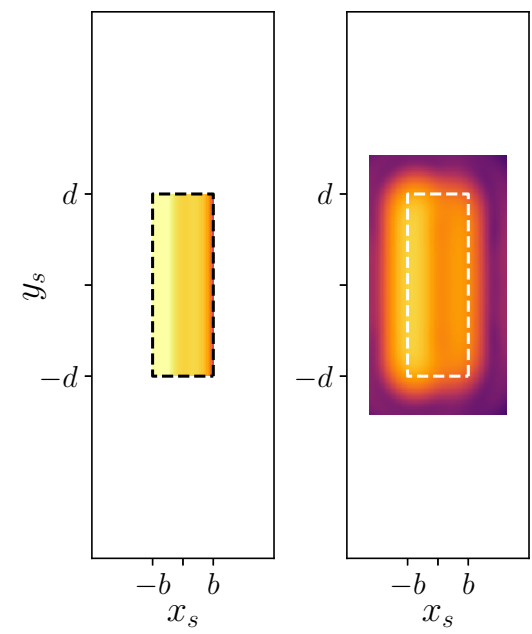

(b)
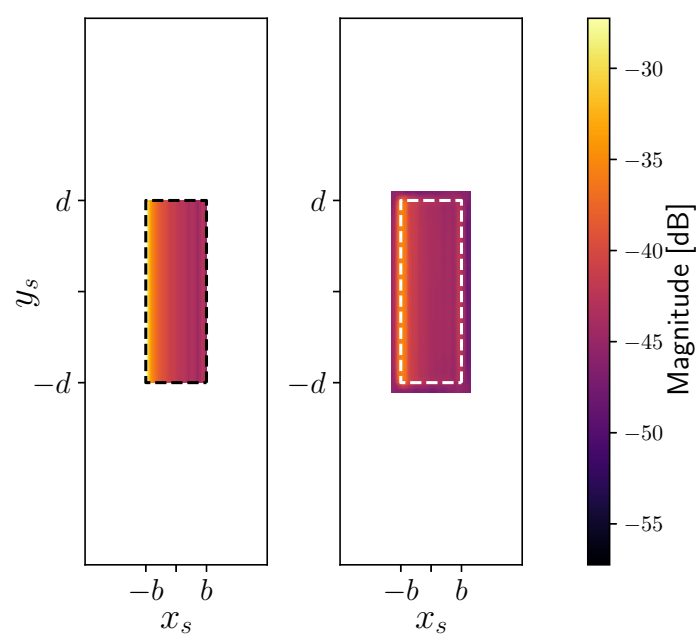

(c)

Figure 6: Original surface pressure PSD magnitude $\left|S_{\Delta p \Delta p}\left(x_{s}, y_{s}\right)\right|$ (left), and equivalent far-field-radiating surface pressure PSD magnitude $\left|S_{\Delta p \Delta p \text {,equiv }}\left(x_{s}, y_{s}\right)\right|$ (right), in $\mathrm{dB}$ : (a) low frequency $\left(k_{0} c=0.5\right)$; (b) medium frequency $\left(k_{0} c=5\right)$; (c) high frequency $\left(k_{0} c=20\right)$. The aerofoil dimensions are indicated with dashed lines, and the dynamic range is $30 \mathrm{~dB}$. 


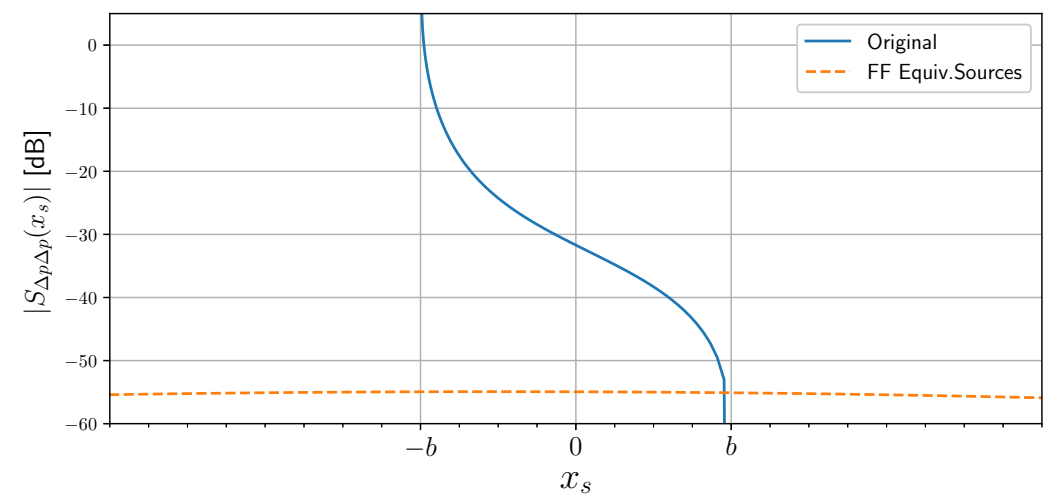

(a)

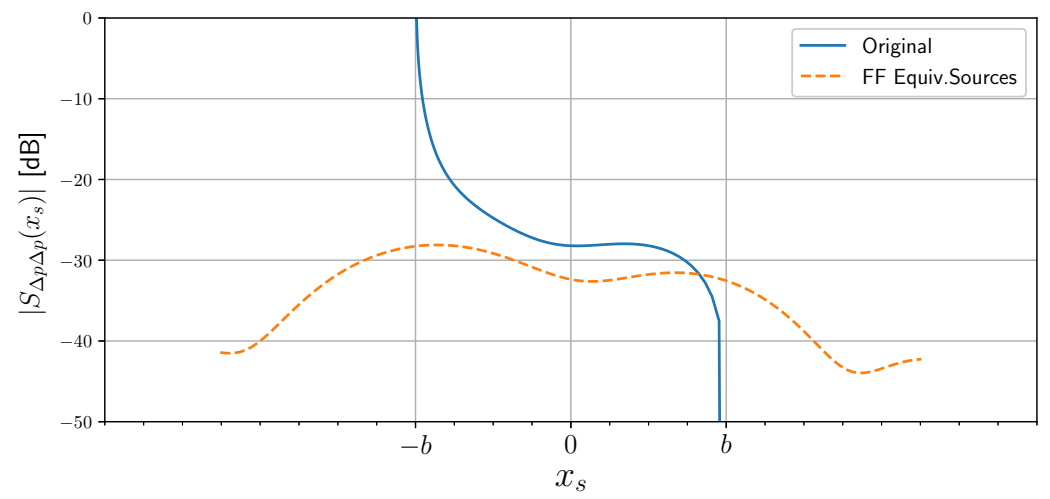

(b)

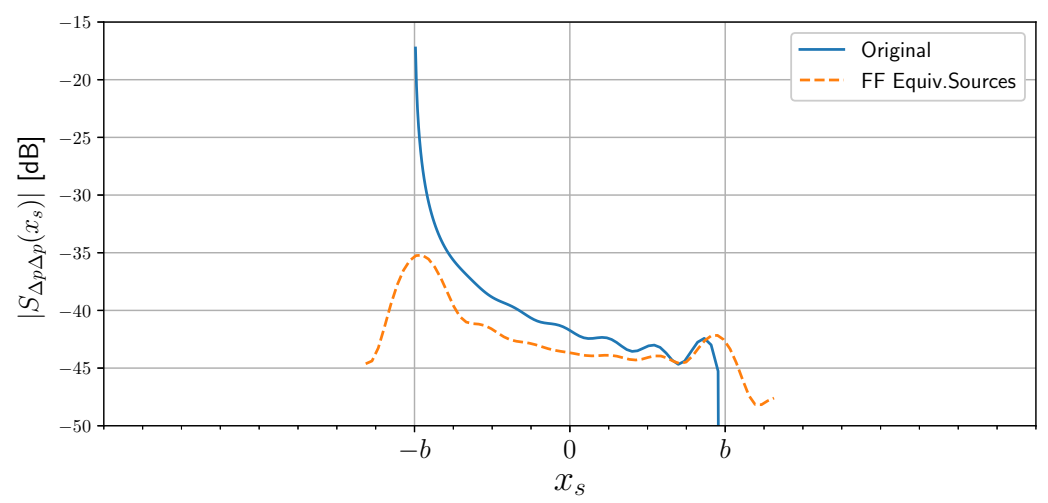

(c)

Figure 7: Chordwise original surface pressure PSD magnitude $\left|S_{\Delta p \Delta p}\left(x_{s}\right)\right|$ (continuous line) and equivalent far-field-radiating surface pressure PSD magnitude $\left|S_{\Delta p \Delta p \text {,equiv }}\left(x_{s}\right)\right|$ (dashed line), evaluated at mid-span $\left(y_{s}=0\right)$ : (a) low frequency $\left(k_{0} c=0.5\right)$; (b) medium frequency $\left(k_{0} c=5\right)$; (c) high frequency $\left(k_{0} c=20\right)$. 


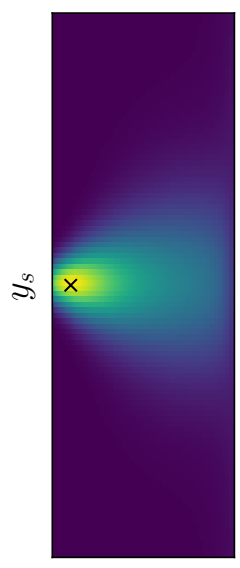

$x_{s}$

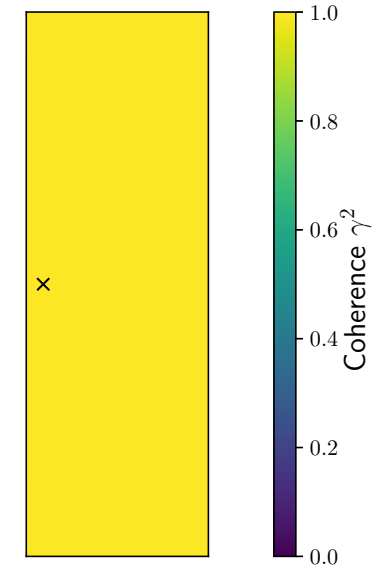

$x_{s}$

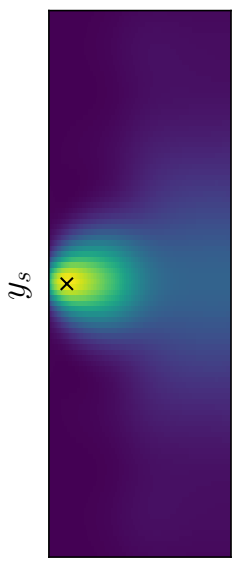

$x_{s}$

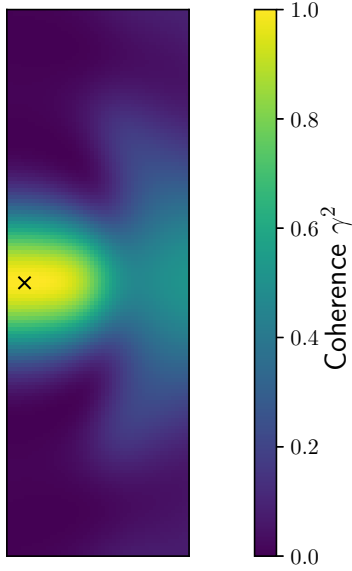

$x_{s}$

(b)

(a)

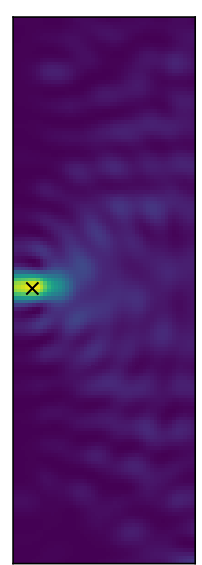

$x_{s}$

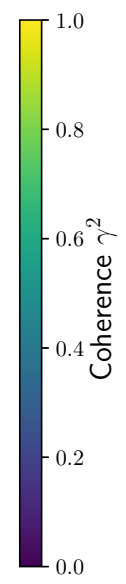

$x_{s}$

(c)

Figure 8: Coherence function $\gamma^{2}\left(\mathbf{r}_{s}, \mathbf{r}_{r e f}\right)$ for original (left) and equivalent far-field-radiating (right) surface pressure, reference point $\mathbf{r}_{r e f}$ indicated with an " $\mathrm{x}$ " marker: (a) low frequency $\left(k_{0} c=0.5\right)$; (b) medium frequency $\left(k_{0} c=5\right)$; (c) high frequency $\left(k_{0} c=20\right)$. 